\title{
Assessment of the self-consistency of electron-THF cross sections using electron swarm techniques: Mixtures of THF-Ar and THF-N2
}

Cite as: J. Chem. Phys. 151, 054309 (2019); https://doi.org/10.1063/1.5108619

Submitted: 30 April 2019 . Accepted: 16 July 2019 . Published Online: 05 August 2019

J. de Urquijo (D), M. J. E. Casey (D), L. N. Serkovic-Loli (D), D. G. Cocks (D), G. J. Boyle (D), D. B. Jones (D), M. J. Brunger (D), and R. D. White (iD)
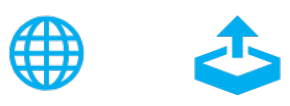

\section{ARTICLES YOU MAY BE INTERESTED IN}

Double Rydberg anions with solvated ammonium kernels: Electron binding energies and Dyson orbitals

The Journal of Chemical Physics 151, 054301 (2019); https://doi.org/10.1063/1.5113614

Sub-nanosecond secondary geminate recombination in mercury halides $\mathrm{HgX}_{2}(\mathrm{X}=\mathrm{I}, \mathrm{Br})$ investigated by time-resolved x-ray scattering

The Journal of Chemical Physics 151, 054310 (2019); https://doi.org/10.1063/1.5096422

Absolute ionization and dissociation cross sections of tetrahydrofuran: Fragmentation-ion production mechanisms

The Journal of Chemical Physics 151, 064304 (2019); https://doi.org/10.1063/1.5115403

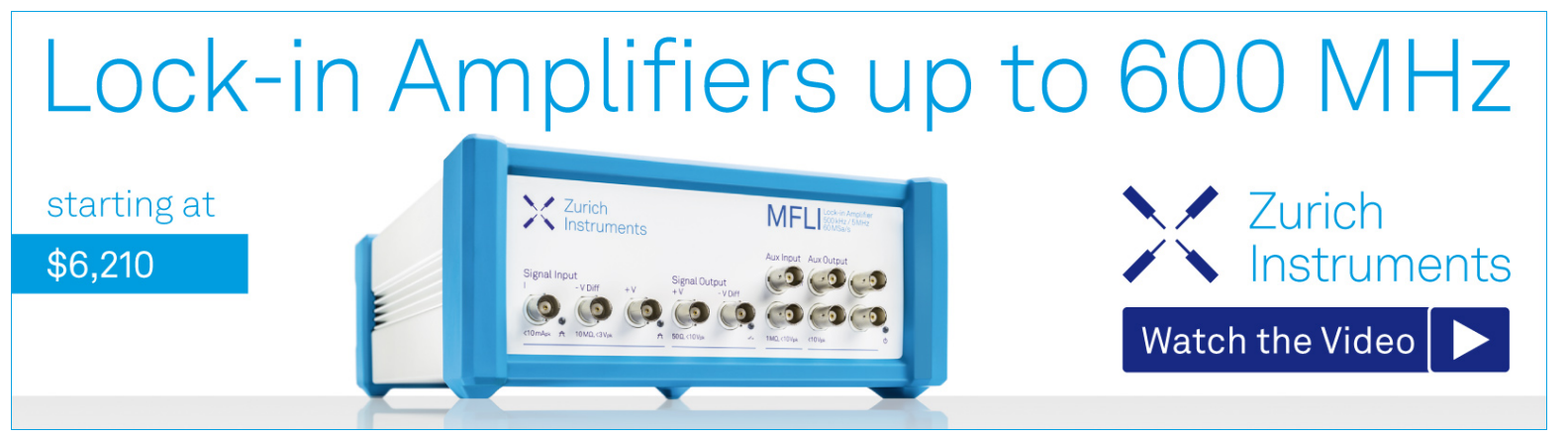




\title{
Assessment of the self-consistency of electron-THF cross sections using electron swarm techniques: Mixtures of THF-Ar and THF-N $\mathbf{N}_{2}$
}

Cite as: J. Chem. Phys. 151, 054309 (2019); doi: 10.1063/1.5108619

Submitted: 30 April 2019 • Accepted: 16 July 2019 •

Published Online: 5 August 2019

J. de Urquijo, (D) M. J. E. Casey, ${ }^{2}$ (D) L. N. Serkovic-Loli, ,a) (D) D. G. Cocks, ${ }^{2, b)}$ (D) G. J. Boyle, ${ }^{2, c)}$ (D)

D. B. Jones, ${ }^{3}$

M. J. Brunger, ${ }^{3}$ (D) and R. D. White ${ }^{2, d)}$ (D)

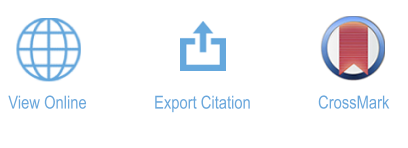

\section{AFFILIATIONS}

${ }^{1}$ Instituto de Ciencias Físicas, Universidad Nacional Autónoma de México, 62251, Cuernavaca, Morelos, Mexico

${ }^{2}$ College of Science and Engineering, James Cook University, Townsville QLD 4811, Australia

${ }^{3}$ College of Science and Engineering, Flinders University, GPO Box 2100, Adelaide SA 5001, Australia

\begin{abstract}
a) Present address: Instituto de Física, Universidad Nacional Autónoma de México, 04510, México City, Mexico.
b) Present address: Research School of Physics and Engineering, The Australian National University, Canberra O200, Australia.

${ }^{c}$ Present address: Linear Accelerator Technologies (FLA), Deutsches Elektronen-Synchrotron (DESY), Notkestraße 85, 22607 Hamburg, Germany.
\end{abstract}

d) Ronald.White@jcu.edu.au

\begin{abstract}
The pulsed Townsend technique has been used to measure transport coefficients in mixtures of tetrahydrofuran (THF) with $\mathrm{N}_{2}$ and argon. These measurements are the first investigations of swarm transport in gas mixtures with THF, with the drift velocity and effective Townsend ionization coefficient reported for a range of reduced electric fields between 0.23 and $800 \mathrm{Td}\left(1 \mathrm{Td}=10^{-21} \mathrm{~V} \mathrm{~m}^{2}\right)$. These transport coefficients are compared with those calculated using a multiterm kinetic theory, using the cross section set developed in our previous studies [N. A. Garland et al., Phys. Rev. A 88, 062712 (2013) and M. J. E. Casey et al., J. Chem. Phys. 147, 195103 (2017)]. The swarm technique of iteratively adjusting cross sections to reproduce experimental transport measurements is subsequently utilized in this study to address the deficiencies in the earlier cross section sets, exposed by the gas mixture measurements. Refinement of the low-energy extrapolation of the quasielastic database cross section and the low-energy extrapolation and magnitude of the dissociative electron attachment cross section are detailed, as well as the adjustments to the two previously proposed neutral dissociation cross sections. These refinements were necessary in order to minimize differences between our measured and calculated transport coefficients.
\end{abstract}

Published under license by AIP Publishing. https://doi.org/10.1063/1.5108619

\section{INTRODUCTION}

Interaction of charged particle species with biological matter is fundamental to understanding energy deposition and induced molecular processes in medical imaging and treatment. In the broad field of plasma medicine, products of plasma interactions are utilized and may be manipulated using electric and magnetic fields, for different purposes, from sterilization to cancer treatment. ${ }^{3-8}$ Modeling of the interaction of plasmas with biological tissue is fundamental for understanding the induced molecular processes and the effects of plasma treatments. ${ }^{8-11}$

The initial, high energy electrons from plasma sources potentially undergo attachment and dissociative processes within the medium but predominantly lose their energy through inelastic interactions, in particular, through ionization events. ${ }^{12}$ These ionization events result in showers of secondary electrons, the most abundant of the secondary species, with greater than $5 \times 10^{4}$ electrons produced for every $\mathrm{MeV}$ of incident radiation, ${ }^{13-1}$ 
typically with energies below $30 \mathrm{eV} \cdot{ }^{13,15,17}$ As the primary and secondary electrons thermalize, the low energy electrons can result in significant molecular damage and potentially lethal lesions to DNA. ${ }^{9-11,18}$ As such, accurate modeling of energy deposition and the production rates of secondary electrons is important for understanding electron transport and damage induced by ionizing radiation.

For the eventual aim of modeling charged particle transport in human tissue, electron interactions with biomolecules are of particular interest. To construct tissue surrogates for representative modeling, simplified targets that are subunits or constituents of DNA and RNA need to be comprehensively studied. Of the many biomolecules under investigation (see, for example, Refs. 19-22), electron scattering from tetrahydrofuran (THF) is one of the most extensively studied, other than water. In the backbone of DNA chains, THF is a sugar connecting the phosphate groups, often used to model the deoxyribose ring.

For swarm and plasma modeling of charged particle transport in simple targets like THF, a complete and accurate description of the microscopic electron-molecule interactions is required in the form of scattering cross sections. ${ }^{23}$ For THF, four full sets of scattering cross sections have been compiled over different energy regimes; see the work of Fuss et al. ${ }^{24}$ for incident electron energies $1 \mathrm{eV}-$ $10 \mathrm{keV}$, Bug et al. ${ }^{20}$ for $30 \mathrm{eV}-1 \mathrm{keV}$ electrons, Swadia et al. ${ }^{25,26}$ for electrons from the ionization threshold to $5 \mathrm{keV}$, and Garland et al. ${ }^{1}$ from $0.1 \mathrm{eV}$ to $300 \mathrm{eV}$. Calculations of swarm transport coefficients using the set of Garland et al. were detailed in that study and in mixtures with $\mathrm{H}_{2} \mathrm{O}$ in the work of White et al., ${ }^{27}$ while the first experimental measurements of swarm transport coefficients in pure THF were presented in the work of Casey et al. ${ }^{2}$ The experimental measurements of the drift velocity and effective Townsend ionization coefficient in the work of Casey et al. allowed for a rigorous assessment of the cross section set of Garland et al. In that study, the swarm inversion technique was employed to iteratively adjust cross sections for some of the individual scattering channels, in particular, those that are difficult to measure via standard crossedbeam methods or to calculate with state of the art scattering theory. This allowed for an optimum gas-phase THF scattering cross section database to be determined.

The swarm technique for unfolding microscopic scattering information has a long history, from the early studies of Huxley, Crompton, Phelps, and co-workers ${ }^{28-31}$ to more recent investigations involving the swarm measurements of the México group. Among the many notable studies advancing the field are those of Haddad and co-workers with argon and mixtures with molecular gases, ${ }^{37-40}$ the studies of Christophorou and co-workers on resolving the cross sections of fluorocarbon gases, among others, ${ }^{41-49}$ and reconciling the errors between experimental and calculated transport in $\mathrm{H}_{2} \mathrm{O}^{50-52}$ by the JCU and México groups, to name but a few. Each of these investigations utilize swarm experiments that measure multiple-scattering events when the electron swarm is in a steadystate with the neutral background gas so that particle, energy, and momentum exchanges during collisions are balanced with the externally applied fields. A complete set of cross sections, either calculated and/or measured during single-scattering experiments, are used to simulate steady-state transport coefficients that are then compared with those from the experimental swarm measurements. The swarm inversion technique involves iteratively adjusting the cross section set until the calculated transport coefficients reproduce experimental values. In a comprehensive treatment, a range of reduced electric fields are used to expose different energy regimes of the cross section set, where different collisional processes dominate the transport. A limitation of the swarm inversion technique is, however, the loss of finer detail ${ }^{28}$ and in resolving the unique set of cross sections for a gas, as discussed specifically for pure THF in the work of Casey et al. ${ }^{2}$ Transport coefficients measured under crossed electric and magnetic fields or in the presence of an admixture of gases, one of which being considered to possess known cross sections, are two of the methods used to reduce the potential nonuniqueness of swarm-derived cross sections. The second of these approaches is utilized in this study, where the presence of a deep Ramsauer minimum in argon and inelastic processes in molecular nitrogen significantly change the energy and momentum exchange at a given electric field, allowing a discriminating test and refinement of the THF cross section set proposed in the work of Casey et al., referred hereafter as I.

The current study is dedicated to assessing and modifying the complete set of cross sections for electron scattering from THF vapor developed in I, using new swarm transport measurements of mixtures with THF, and is arranged as follows. In Sec. II, the experimental technique describing the pulsed Townsend apparatus, used to measure the current trace for an electron swarm in gas mixtures with THF, is outlined. The connection between the theoretically calculated transport coefficients and those extracted from the experimental conditions are discussed briefly in Sec. III A, along with our multiterm Boltzmann equation solution in Sec. III B. Measurements of the drift velocity and effective Townsend ionization coefficient in mixtures of THF with argon and $\mathrm{N}_{2}$ are reported in Sec. IV A and tabulated in the supplementary material. In Sec. IV B 1, the complete set of THF electron scattering cross sections compiled in I is outlined, with the cross section sets for both argon and $\mathrm{N}_{2}$ used for the mixture calculations in this study detailed in the Appendix. Using these cross sections, our calculated transport coefficients are compared with our experimental measurements in Sec. IV B 2. Thereafter, in Sec. IV B 3, the modifications to the THF cross section set required to better reproduce the experimental mixture measurements are discussed, and the resulting transport coefficients are detailed. Finally, in Sec. V, some final remarks on the results of this study are drawn.

\section{EXPERIMENTAL TECHNIQUE}

The measurements presented in this study for the mixtures of THF vapor with argon and $\mathrm{N}_{2}$ were derived from analysis of electron avalanches measured with a fully automated pulsed Townsend apparatus. The experimental technique and resulting transport coefficients have been described previously; ${ }^{53,54}$ thus, we only outline the relevant details again here. The initial photoelectrons impinging upon the cathode are produced by a $3 \mathrm{~ns}$-duration laser $(355 \mathrm{~nm}$, $1 \mathrm{~Hz}$ repetition rate). These electrons and their progeny resulting from collisions with the gas molecules are driven by an external electric field toward the anode. The total displacement current due to electron and ion motion is collected and analyzed. A highly stable voltage (between 0.2 and $5 \mathrm{kV}$ ) was applied between the plates to produce a highly homogeneous DC electric field $E$, according 
to the selected $E / n_{0}$ for the neutral gas density $n_{0}$ in the discharge vessel. The parallel-plate capacitor consists of an aluminum cathode and a nonmagnetic stainless steel anode of $12 \mathrm{~cm}$ diameter each. The gap distance was kept fixed at $3.1 \mathrm{~cm}$ to an accuracy of $0.025 \mathrm{~mm}$.

When the dominant processes are electron and ion motion and the ionization and attachment processes are due only to collisions of electrons with the gas molecules, the measured current can be separated into a fast electron component and a slower component due to ion motion. For mixtures involving the weakly attaching THF gas, the current trace was readily separable into these two components. The displacement current due to the electrons was measured with a low-noise, $40 \mathrm{MHz}$ amplifier with a transimpedance of $10^{5} \mathrm{~V} / \mathrm{A}$. Analysis of the current trace for the derivation of the electron drift velocity, $W$, and the macroscopic effective Townsend ionization coefficient, $\alpha_{\text {eff }} / n_{0}$, have been described elsewhere, and the reader is referred to Hernández-Ávila et al. ${ }^{5}$ and Basurto et al. ${ }^{54}$ for the details. Here, $\alpha_{\text {eff }}=\alpha_{T}-\eta_{T}$, where $\alpha_{T}$ and $\eta_{T}$ are the macroscopic ionization and attachment coefficients, respectively.

The purity of THF as stated by the manufacturer was $99.9 \%$ (Sigma Aldrich), whereas that for $\mathrm{Ar}$ and $\mathrm{N}_{2}$ was $99.95 \%$ (Praxair). The measurements were performed at room temperatures between 293 and $300 \mathrm{~K}$ and measured with a precision of $\pm 0.5 \mathrm{~K}$. The pressure in the discharge vessel was measured with an absolute pressure capacitance gauge with a manufacturer's stated accuracy of $0.15 \%$.

Provided that the saturation vapor pressure of THF is 143 Torr at $293 \mathrm{~K}$, our measurements were carried out well below this value in order to avoid any vapor condensation effects in the gas discharge vessel walls. As a matter of fact, the maximum THF pressure in the vessel was 40 Torr. The THF-X gas mixtures, where $\mathrm{X}$ is either $\mathrm{Ar}$ or $\mathrm{N}_{2}$, were carefully set by first injecting the minority gas and then replenishing with $\mathrm{Ar}$ or $\mathrm{N}_{2}$ until the gas mixture was set with an uncertainty of $0.3 \%$. The drift velocity measurements are accurate to within $2 \%-3 \%$ over the whole range of $E / n_{0}$ and gas mixture fractions, while uncertainties in the effective ionization coefficient lie within $6 \%-10 \%$. It is to be noted that the values of $\alpha_{\text {eff }} / n_{0}$ for the mixtures with 1\%-5\% THF (see the lower panel of Fig. 9) in Ar must be taken as only trends since $10^{-24} \mathrm{~m}^{2}$ is our lower measurement limit at low $E / n_{0}$ since the amplitude of the signal is very small due to the fairly long electron transit times, $T_{e}$, across the discharge gap. As a matter of fact, the displacement current, $I_{e}$, is inversely proportional to $T_{e} \cdot{ }^{53,54}$ The low $E / n_{0}$ region is usually the most difficult one to measure since low gap voltages are used $(\geq 200 \mathrm{~V})$ but not so low as to become compatible to the space charge field caused by the avalanche cloud itself.

\section{THEORY}

\section{A. Transport coefficients and the pulsed Townsend experiment}

Two sets of transport coefficients represent the behavior of parts of the swarm-the bulk and flux coefficients. The bulk coefficients represent the average transport of the center of mass in configuration space, while the flux coefficients represent averages over the swarm ensemble in velocity space. The particular coefficients extracted from experiments are often dependent on how the measurements are analyzed. ${ }^{55}$ For example, for time-of-flight experiments, the diffusion equation, $\frac{\partial n(\mathbf{r}, t)}{\partial t}+\mathbf{W}_{B} \cdot \nabla n-\mathbf{D}_{B}: \nabla \nabla n$ $=n R_{\text {net }}(t)$, is used to extract the bulk coefficients, that define the bulk drift velocity $\mathbf{W}_{B}$, the bulk diffusion tensor $\mathbf{D}_{B}$, and the nett particle loss rate $R_{\text {net }}$, of the electron swarm with number density $n$ at a position $\mathbf{r}$ and time $t$. The flux coefficients, on the other hand, are extracted from an analysis of pulsed Townsend experiments where they are defined by the use of a number density gradient expansion employing Fick's Law. ${ }^{56}$ In the absence of particle nonconserving processes, like ionization or attachment, the bulk transport coefficients reduce to the flux coefficients.

In the pulsed Townsend experiment, near the cathode large gradients exist, and in the presence of particle nonconserving processes, the behavior of the swarm is nonhydrodynamic, so a steadystate is reached when the transport coefficients are constant in time but may vary in configuration space. However, sufficiently far from the source cathode, away from the steep density gradients and in the long time limit, the electron velocity distribution reaches a steady-state. Here, hydrodynamic conditions prevail and the pulsed Townsend experiment may be analyzed in terms of the equation of continuity for the number density, $n(\mathbf{r}, t)$,

$$
\frac{\partial n(\mathbf{r}, t)}{\partial t}+\nabla \cdot \boldsymbol{\Gamma}(\mathbf{r}, t)=S(\mathbf{r}, t)
$$

providing the connection with the experimentally measurable swarm transport coefficients. A density gradient expansion of the charged particle flux, using Fick's Law,

$$
\boldsymbol{\Gamma}(\mathbf{r}, t)=n \mathbf{W}-\mathbf{D} \cdot \nabla n(\mathbf{r}, t)+\ldots,
$$

defines the flux coefficients as the constants of proportionality of the number density. Here, $\mathbf{W}$ is the flux drift velocity and the flux diffusion tensor is defined by $\mathbf{D}=D_{T} \mathbf{I}+\left(D_{L}-D_{T}\right) \hat{\mathbf{E}} \hat{\mathbf{E}}$, where $\mathbf{I}$ is the unit tensor and $\hat{\mathbf{E}}$ is a unit vector in the direction of $q \mathbf{E}$, where $\mathbf{E}$ is the applied external field and $q$ is the elementary charge. The flux diffusion tensor separates into time dependent scalar quantities, the transverse $\left(n_{0} D_{T}\right)$, and longitudinal $\left(n_{0} D_{L}\right)$ diffusion coefficients.

In the pulsed Townsend experiment, the assumed form of the number density gradient expansion and the definition of the effective Townsend ionization coefficient for the source term in Eq. (1), $S(\mathbf{r}, t)$, combine so that the current trace of the experiment is assumed to take the approximate form detailed in Refs. 52, 56, and 57.

In the hydrodynamic regime, it is assumed that the number density is given by $n(z) \sim \exp \left(\alpha_{\text {eff }} z\right)$, where $\alpha_{\text {eff }}$ defines the macroscopic effective Townsend ionization coefficient and $z$ is the spatial coordinate taken to be perpendicular to the electrodes. The steadystate Townsend transport coefficients, denoted by the subscript SST, are related to the flux quantities through

$$
\begin{aligned}
\langle\epsilon\rangle_{S S T} & =\varepsilon+\alpha_{\text {eff }} \gamma+\cdots, \\
W_{S S T} & =W-\alpha_{\text {eff }} D_{L}+\cdots .
\end{aligned}
$$

In the absence of particle nonconserving processes, the steady-state Townsend coefficients also reduce to the flux transport coefficients. Of particular interest in this study is the macroscopic effective 
Townsend ionization coefficient $\alpha_{\text {eff }} / n_{0}$ that is related to the nett reaction rate $R_{\text {net }}$ by

$$
R_{\mathrm{net}}=\alpha_{\mathrm{eff}} W_{B}-\alpha_{\mathrm{eff}}^{2} D_{L, B}+\cdots
$$

where $R_{\text {net }}$ involves the bulk transport coefficients, as given in Refs. 55 and 58 .

\section{B. Multiterm solution of the Boltzmann equation and calculated transport coefficients}

Calculation of the transport coefficients for comparison with the experimentally measurable parameters requires the phase-space distribution function $f(\mathbf{r}, \mathbf{v}, t)$ describing the swarm of electrons in configuration space $\mathbf{r}$, with velocity $\mathbf{v}$ and time $t$. The interactions between the swarm and neutral background gas can be described by the Boltzmann equation

$$
\left(\frac{\partial}{\partial t}+\mathbf{v} \cdot \nabla+\frac{q \mathbf{E}}{m_{e}} \cdot \frac{\partial}{\partial \mathbf{v}}\right) f(\mathbf{r}, \mathbf{v}, t)=-J(f(\mathbf{r}, \mathbf{v}, t)),
$$

where $m_{e}$ is the mass of the swarm particle. The right-hand side describes all of the electron-neutral collisions via the linear Boltzmann collision operator $J$, which accounts for all of the microscopic interactions in each component $i$ of the gas mixture, where $J(f)=\sum_{i} \alpha_{i} J_{i}(f)$, for molar fractions of the gas components $\alpha_{i}$.

To solve Eq. (6) for the phase-space distribution function, the angular dependence is expanded in terms of spherical harmonics $f(\mathbf{r}, \mathbf{v}, t)=\sum_{l=0}^{\infty} \sum_{m=-l}^{l} f_{l, m}(\mathbf{r}, v, t) Y_{m}^{[l]}(\hat{v})$. In practice, the $l$ index is truncated at some value $l=l_{\max }$, when some convergence criterion on the distribution function or its velocity moments is satisfied. In this manner, our solution is a multiterm solution that is not limited to the two-term approximation that restricts the simulation of the anisotropic distortion of the electron swarm. ${ }^{59}$ To represent the steady-state Townsend experimental configuration, the spatial gradients are perpendicular to the electrodes, taken along the $z$-axis so that $\mathbf{r}=z$ and $\mathbf{E}=E_{z}$. Symmetry allows the $m$ index to be truncated at $m=0$ so that application of the spherical harmonic expansion in plane parallel geometry results in the following hierarchy:

$$
\begin{aligned}
\frac{\partial f_{l}}{\partial t} & +J_{l}\left(f_{l}\right)+\left(\frac{2 q}{m_{e}}\right)^{1 / 2} \sum_{p= \pm 1} \Delta_{l}^{(p)}\left[U^{1 / 2} \frac{\partial}{\partial z}\right. \\
& \left.+E_{z}\left(U^{1 / 2} \frac{\partial}{\partial U}+\frac{p}{2}\left(l+\frac{3 p+1}{2}\right) U^{-1 / 2}\right)\right] f_{l+p}=0
\end{aligned}
$$

where

$$
\begin{aligned}
& \Delta_{l}^{(+)}=\frac{l+1}{2 l+3}, \\
& \Delta_{l}^{(-)}=\frac{l}{2 l-1},
\end{aligned}
$$

that has been recast in energy space for $U$ in eV using $q U=\frac{1}{2} m_{e} v^{2}$. The collision operator $J_{l}$ describes the binary interactions for each collision type for each component of the gas mixture $i$, where $J_{l}\left(f_{l}\right)=\sum_{i} \alpha_{i}\left(J_{l, i}^{\text {elas }}\left(f_{l}\right)+J_{l, i}^{\text {inel }}\left(f_{l}\right)+J_{l, i}^{\text {att }}\left(f_{l}\right)+J_{l, i}^{\text {ion }}\left(f_{l}\right)\right)$. The superscripts refer to the collision operators for elastic collisions, ${ }^{60-62}$ particle-conserving inelastic collisions, ${ }^{28,63-65}$ particle-loss attachment, and particle-gain ionizing collisions, ${ }^{66}$ respectively. Each of these has been described in detail in the respective Refs. 28 and 60-66, and where necessary the methods for treating collisions described in Ref. 67 have been utilized.

For the present study, where we compare experimentally measured and simulated drift velocities and the effective Townsend ionization coefficient, we calculate time-of-flight and steady-state Townsend coefficients for each of these, respectively. For details, the reader is referred to the approaches used in the work of Casey et al. ${ }^{67}$ and Boyle et al. ${ }^{68,69}$ that have been systematically benchmarked and validated against independent kinetic theory solutions and Monte Carlo methods.

The solution to Eq. (7) is the required distribution function $f_{l}(z, U, t)$, moments of which yield the measurable transport coefficients of the swarm. For the coefficients of interest in this work, the spatial dependence of the distribution function is represented using a density gradient expansion when weak-gradient, hydrodynamic, conditions prevail. In plane parallel geometry, the density gradient expansion of the distribution function to second order is given by:

$$
\begin{aligned}
f_{l}(z, U, t)= & F_{l}(U) n(z, t)-F_{l}^{(L)}(U) \frac{\partial n(z, t)}{\partial z} \\
& +\sqrt{\frac{1}{3}} F_{l}^{(2 T)}(U) \frac{\partial^{2} n(z, t)}{\partial z^{2}}-\sqrt{\frac{2}{3}} F_{l}^{(2 L)}(U) \frac{\partial^{2} n(z, t)}{\partial z^{2}} .
\end{aligned}
$$

Employing this expansion, the solutions to Eq. (7) are the $F_{l}^{(s)}$ hydrodynamic density gradient expansion coefficients used to calculate the measurables of interest in this study. Namely, the mean energy $\langle\varepsilon\rangle$, the bulk drift velocity $W_{B}$, the nett rate coefficient $R_{\text {net }}$ summed over all reactive collision frequencies $v_{0}^{R}(U)$, and the longitudinal bulk diffusion coefficient $D_{L, B}$, which are defined by

$$
\begin{aligned}
\langle\varepsilon\rangle= & 2 \pi q\left(\frac{2 q}{m_{e}}\right)^{3 / 2} \int U^{3 / 2} F_{0}(U, t) d U, \\
W_{B}= & \frac{2 \pi}{3}\left(\frac{2 q}{m_{e}}\right)^{2} \int U F_{1}(U, t) d U \\
& -2 \pi\left(\frac{2 q}{m_{e}}\right)^{3 / 2} \int U^{1 / 2} J_{0}^{R}\left(F_{0}^{(L)}(U, t)\right) d U, \\
R_{\text {net }}= & \sum_{R} 2 \pi\left(\frac{2 q}{m_{e}}\right)^{3 / 2} \int U^{1 / 2} v_{0}^{R}(U) F_{0}(U, t) d U, \\
D_{L, B}= & \frac{2 \pi}{3}\left(\frac{2 q}{m_{e}}\right)^{2} \int U F_{1}^{(L)}(U, t) d U-2 \pi\left(\frac{2 q}{m_{e}}\right)^{3 / 2} \\
& \times \int U^{1 / 2} J_{0}^{R}\left(\frac{1}{\sqrt{3}}\left[F_{0}^{(2 T)}(U, t)-\sqrt{2} F_{0}^{(2 L)}(U, t)\right]\right) d U,
\end{aligned}
$$

where $J^{R}$ is the total reactive collision operator. The flux coefficients are calculated using the first term of the $W_{B}$ and $D_{L, B}$ coefficient definitions. The effective Townsend ionization coefficient can be calculated using these hydrodynamic transport coefficients through the expansion (5) or directly through simulation of the steady-state Townsend coefficients. 


\section{RESULTS AND ANALYSIS}

\section{A. Pulsed Townsend measurements of the drift velocity and the effective Townsend ionization coefficient in THF-Ar and THF- $\mathrm{N}_{2}$ mixtures}

The focus of this study is the pulsed Townsend measurements of the drift velocity and effective Townsend ionization coefficient for admixtures of THF with argon and $\mathrm{N}_{2}$. In Figs. 1 and 2 and the supplementary tables, we present our measurement data at varying mixture fractions as a function of the reduced electric field in units of the Townsend $\left(1 \mathrm{Td}=10^{-21} \mathrm{~V} \mathrm{~m}^{2}\right)$. Measurements of the electron swarm transport coefficients in both of the pure gases are deferred to the Appendix.

At the lower $E / n_{0}$ range of our experimental measurements, the argon and THF mixtures have higher drift velocities than both of the pure gas profiles. The argon mixtures with lower proportions of THF have larger drift velocities than the higher admixtures of THF as might be expected from the stronger magnitude of the pure argon profile as compared to the pure THF profile at the same $E / n_{0}$. The
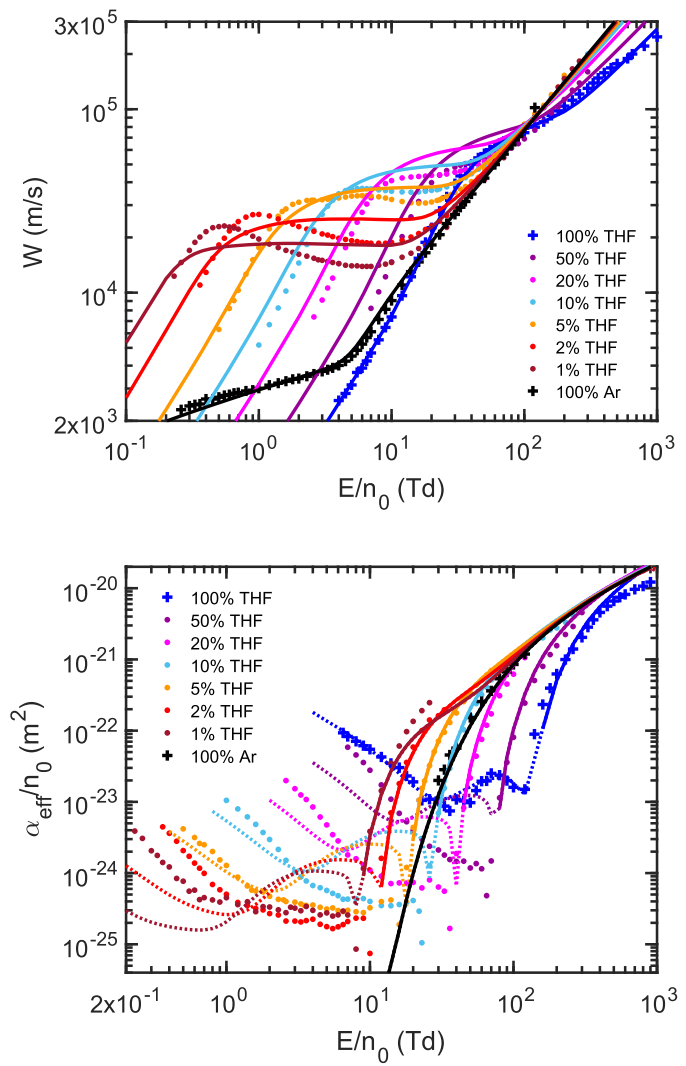

FIG. 1. (Upper) The electron drift velocity and (lower) the effective Townsend ionization coefficient in THF-Ar mixtures as a function of the reduced electric field $E / n_{0}$. The symbols correspond to our measurements, while the lines represent our calculated values using the THF cross section set proposed in I. The broken lines correspond to the electronegative region of the effective Townsend ionization coefficient with the change to ionization-dominated transport denoted by the solid lines.

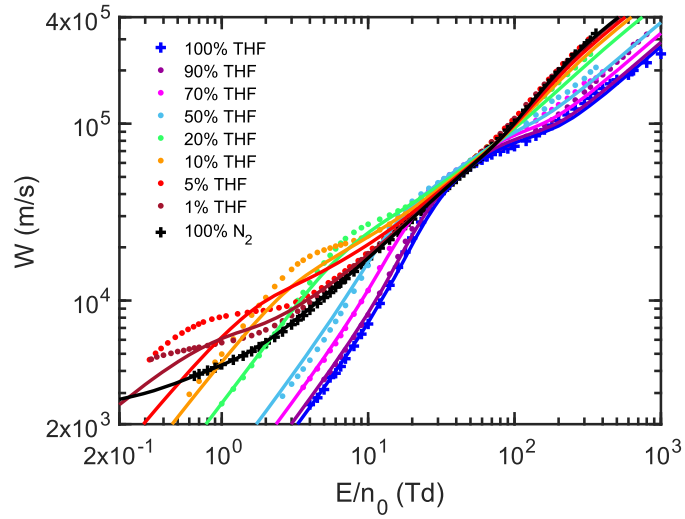

FIG. 2. The electron drift velocity in THF- $\mathrm{N}_{2}$ mixtures as a function of the reduced electric field $E / n_{0}$. The symbols correspond to our measurements, while the lines represent our calculated values using the THF cross section set proposed in I.

small addition of $1 \%$ THF to argon results in a significant change to the drift velocity profile from that for pure argon. The quasielastic ${ }^{70}$ momentum transfer cross section of THF is much larger than that of argon, between one and two orders larger below $1 \mathrm{eV}$ (compare the "current" argon elastic momentum-transfer profile in Fig. 1 of Boyle et al. ${ }^{69}$ with the THF quasielastic momentum-transfer profile in Fig. 3). Combined with the multiple excitations of THF with lowenergy thresholds, the small addition of THF modifies the energy distribution by reducing the mean energy of the swarm to near thermal values to sample lower-energy regions of the cross sections, resulting in a larger drift velocity than for pure argon. The increasing drift velocity at lower $E / n_{0}$ is suppressed when the THF electronicstate channels open in the THF-Ar mixtures. At the onset of these electronic-state excitations, the drift velocity reduces with increasing $E / n_{0}$, resulting in a negative differential conductivity (NDC) region, in all of the THF-Ar mixture fractions. This feature is absent from both of the pure-gas drift velocity profiles. This phenomenon is well

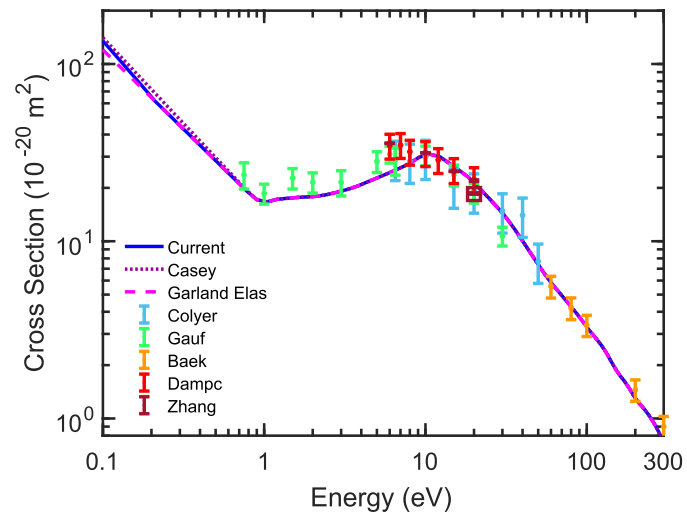

FIG. 3. The proposed quasielastic momentum-transfer cross section for electron impact on THF, compared with the cross section of I, the (elastic only) estimate of Garland et al. ${ }^{1}$ and the experimental cross sections of Colyer et al., ${ }^{80}$ Gauf et al., Baek et al., ${ }^{82}$ Dampc et al.., and Zhang et al. ${ }^{84}$ (the dots based on Dampc et al. and the square based on Colyer et al.). 
known and described in Refs. 32, 33, 52, and 71. As the reduced electric field increases further and the higher-threshold excitation channels and the ionization cross sections start to dominate the transport, the drift velocity profiles for all the THF-Ar mixture fractions approach each other, falling between the two pure gas profile cases.

In the absence of an attachment process in argon, at the lower $E / n_{0}$, and before the opening of any ionization channel, the magnitude of the effective Townsend ionization coefficient increases toward that of the pure THF profile with increasing amounts of THF in the mixture composition. Compared to the pure THF attachment rate, there is, however, a change in the rate at which $\alpha_{\text {eff }} / n_{0}$ decreases with increasing $E / n_{0}$, in the attachment-dominated region, as exhibited by all of the mixture ratios. In the mixture measurements, there is also an absence, or significant suppression, of the "resonance" region, i.e., where an increase in the attachment-dominated part of $\alpha_{\text {eff }} / n_{0}$ is identified before the change to ionization-dominated transport. In the pure THF profile, this resonance was identified by an increase in the electronegative component of the $\alpha_{\text {eff }} / n_{0}$ from $35 \mathrm{Td}$, before decreasing at $70 \mathrm{Td}$, followed by a change to ionizationdominated transport at $120 \mathrm{Td}$. The ionization-dominated part of $\alpha_{\text {eff }} / n_{0}$ for both pure gases increases at a similar rate (although the onset occurs at different fields, but with similar mean energies according to our calculations) so that the ionization-dominated part of all of the THF-Ar mixture measurements is similar in the profile. The ionization-dominated part of the $\alpha_{\text {eff }} / n_{0}$ profiles shows, at a given $E / n_{0}$, a higher rate of ionization for decreasing amounts of THF in the mixture fractions. At the lower THF admixture fractions, this rate decreases below that in pure argon due to the change in the average energy of the swarm from the THF mixtures.

Finally, we also note that in the attachment-dominated region, the 1\% THF-99\% Ar mixture profile shows different behavior to the other mixture fractions, where the attachment part of the profile increases in magnitude to be larger than that in the $2 \%$ THF admixture profile. We discuss the possible source of this behavior in Sec. IV B 3.

Considering Fig. 2, we find that in the mixtures of THF and $\mathrm{N}_{2}$, the drift velocity increases linearly with increasing $E / n_{0}$, indicative of an essentially thermal energy distribution. A small suppression in the rate of increase in the drift velocity, with increasing $E / n_{0}$, is observed, corresponding to the opening of the $\mathrm{N}_{2}$ vibrational modes. We note that this occurs at much lower reduced electric fields for the $1 \%$ and $5 \%$ THF admixtures, compared to the higher THF admixture ratios with $\mathrm{N}_{2}$. From approximately $30-60 \mathrm{Td}$, the two pure vapor profiles coincide and the variation in the measured drift velocities for all mixtures is suppressed. At higher $E / n_{0}$, the higher-threshold excitation channels and ionization cross sections of THF and $\mathrm{N}_{2}$ start to dominate the transport, resulting in the mixture drift velocities falling between those for the two pure gas profiles for mixtures with $\geq 10 \%$ THF and above both pure profiles for the $1 \%$ and $5 \%$ THF admixture fractions.

\section{B. Comparison of the measured and calculated transport coefficients and self-consistency of the cross section set}

To start our assessment of the completeness and accuracy of the currently preferred THF cross section set, we compare our measured transport coefficients with those calculated using the set of cross sections developed in our previous study. ${ }^{2}$ That set is summarized below with the details of the $\mathrm{Ar}$ and $\mathrm{N}_{2}$ scattering cross section sets employed in this investigation and comparison of the resulting transport coefficients with measurement described in the Appendix.

\section{THF base cross section set}

The cross section set developed in I, labeled in the following figures as "Casey," was adapted from the study of Garland et al. ${ }^{1}$ to reproduce the pure THF experimental transport coefficients detailed in I. That set of cross sections encompass the following:

- A quasielastic momentum-transfer cross section developed in the work of Garland et al., ${ }^{1}$ based on experimental measurements, which contains contributions from elastic, rotational, and possibly some vibrational scattering. Discrimination into separate elastic and rotational scattering channels could not be assessed with the available swarm measurements. Modifications to the quasielastic cross section of Garland et al. below the range of the experimental measurements, but within the experimental error bars, were required at energies below $1 \mathrm{eV}$, as is shown in Fig. 3 .

- The revised and extended vibrational cross sections of Duque et al. ${ }^{72}$ were adopted in order to increase the total magnitude of the vibrational scattering channels through 12 discrete cross sections with a range from the threshold up to $50 \mathrm{eV}$.

- The six electronic-state cross sections estimated in the work of Garland et al., ${ }^{1}$ based on the energy-loss spectra of Do et al., ${ }^{73}$ were maintained.

- The dissociative electron attachment (DEA) cross section proposed in I and shown in Fig. 4, which is the combination of the experimental measurements of Aflatooni et al. and Janečková et al., ${ }^{75}$ with a lower-energy extrapolation, was adopted for our initial calculations. The DEA cross section below $4 \mathrm{eV}$, as adopted from the measurements of

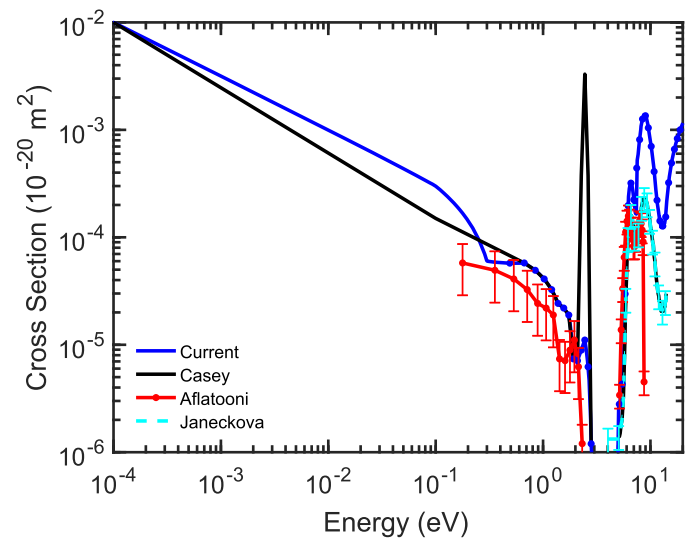

FIG. 4. The proposed dissociative electron attachment cross section (blue), compared with the DEA cross section proposed in I (black), constructed from the experimental total DEA cross sections of Aflatooni et al. ${ }^{74}$ (red) and Janečková et al. ${ }^{75}$ (cyan). 
Aflatooni et al., was shifted in energy by $360 \mathrm{meV}$ in I, consistent with the results of Janečková et al. at the higher energies. The large increase in this cross section proposed around $2.5 \mathrm{eV}$ in $\mathrm{I}$, to reproduce the "resonance" feature in the experimental $\alpha_{\text {eff }} / n_{0}$ at around 35-120 Td, was included and its consistency with the mixture measurements is assessed in Sec. IV B 3 b. We note that this cross section likely represents a total attachment cross section, including the dissociative electron attachment component, although we will maintain the notation "DEA" throughout this study.

- The ionization cross section of Możejko and Sanche, ${ }^{76}$ with the experimental threshold of $9.55 \mathrm{eV}$ taken from Dampc et al., was adopted in the work of both Garland et al. and I.

- Significant modifications to the previously derived neutral dissociation cross sections in the literature were proposed in I, to reproduce the pure THF experimental transport coefficients, and are shown in Fig. 5. The lower, $1 \mathrm{eV}$ threshold, neutral dissociation cross section of Garland et al. was adapted with a magnitude reduced to $10 \%$ of the original and a larger energy spread from $1 \mathrm{eV}$ to $10 \mathrm{eV}$. This was needed to reproduce the shoulder region (at the onset of the electronic-state excitation channels) in the drift velocity measurements. Additionally, the higher-threshold neutral dissociation cross section proposed in the study of Fuss et al. ${ }^{78}$ was also adapted, with the threshold lowered to $10 \mathrm{eV}$, to largely reproduce the change from attachment- to ionization-dominated transport in the effective Townsend coefficient.

- The quasielastic integral cross section (ICS) proposed in I and shown in Fig. 6 was produced to be consistent with the grand total cross section of the Madrid group. ${ }^{24,79}$

\section{Comparison of transport coefficients for the THF admixtures}

For a cross section set that is self-consistent, it must be both accurate and complete in order to reproduce transport coefficients

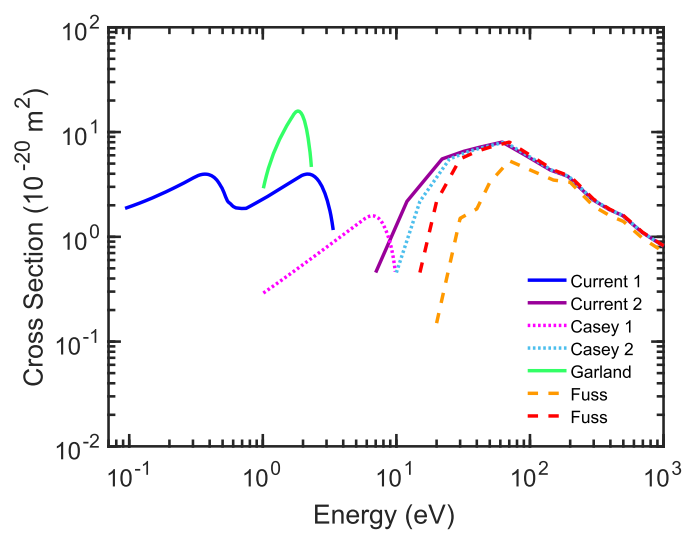

FIG. 5. The two proposed neutral dissociation ICS, compared with the two neutral dissociation processes proposed in I to largely reproduce the pure THF measurements, compared with the remnant Garland et al. ${ }^{1}$ and Fuss et al. ${ }^{24,78}$ neutral dissociation cross sections.

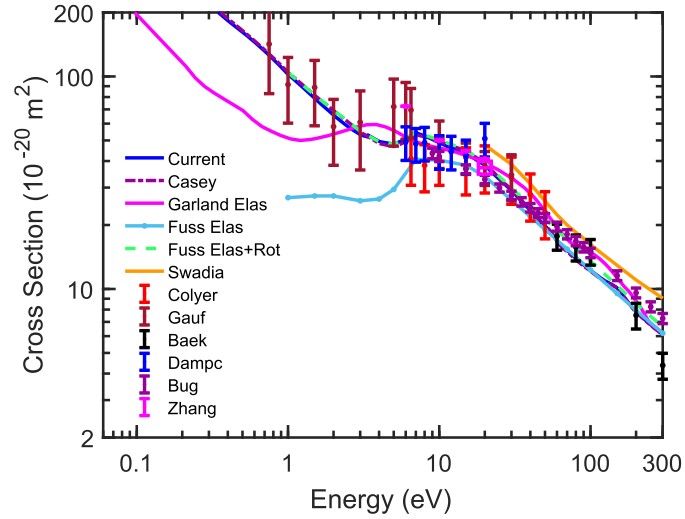

FIG. 6. The quasielastic integral cross section for electron impact on THF proposed to reproduce the grand total cross section of Fuss et al., ${ }^{24}$ with the modifications discussed in Sec. IV B 3. This cross section is compared with the cross section of I and Garland et al. ${ }^{1}$ (elastic only), and the measurements and calculations of Fuss et al. ${ }^{24}$ with and without rotations, Swadia et al., ${ }^{25,26}$ Colyer et al., ${ }^{80}$ Gauf et al.,, Baek et al., ${ }^{82}$ Dampc et al.,, ${ }^{83}$ Bug et al.,, ${ }^{20}$ and Zhang et al. ${ }^{84}$ (the dots based on Dampc et al. and the square based on Colyer et al.).

measured under any experimental conditions that can be simulated. Using the cross section set developed in I, we have calculated the transport coefficients of interest in mixtures of THF with argon and $\mathrm{N}_{2}$. Shown in Figs. 1 and 2 are the results from these simulations, and when compared to our present experimental measurements, we find some inconsistencies which are now described.

For all of the THF-Ar mixtures and the $\geq 20 \%$ fractions of THF in mixtures with $\mathrm{N}_{2}$, the calculated drift velocities at low energies and fields somewhat overestimate our drift velocity measurements. The original underestimation in this $E / n_{0}$ region of the drift velocity, in the pure THF measurements, was specifically addressed by adjustment of the extrapolation of the quasielastic cross section to very low energies. ${ }^{2}$ At the lower THF admixture fractions with $\mathrm{N}_{2}, 1 \%, 5 \%$, and $10 \% \mathrm{THF}$, the calculated drift velocities are now found to be much lower than our measured values and do not show the same qualitative discrepancies as the higher THF fractions in $\mathrm{N}_{2}$ results and all of the THF-Ar mixture results.

When the THF electronic excitation channels open, the rate of increase in the drift velocity with $E / n_{0}$ is suppressed in the THF-Ar measurements, resulting in an NDC region that is not reproduced by our mixture calculations. Here, the qualitative shape of a local maximum, and at higher fields a local minimum, displayed by all mixture fractions in the measurements, is mostly averaged over with an almost constant drift velocity calculated in that $E / n_{0}$ region. In this $E / n_{0}$ region, for the drift velocity in pure $\mathrm{THF},{ }^{2}$ our calculations were originally guided by the adjustment of a low threshold excitation taken to be due to a neutral dissociation process. We also note, in the THF- $\mathrm{N}_{2}$ measurements, the suppression of the drift velocity at the intermediate $E / n_{0}$ corresponds to the opening of the $\mathrm{N}_{2}$ vibrational modes.

At the higher reduced electric fields, where the ionization processes start to dominate the transport, the measured drift velocities 
in both mixture sets are generally underestimated by our calculations as may be expected given the underestimation of our pure drift velocity measurements by our calculations in THF, argon, and $\mathrm{N}_{2}$.

Comparing our measured and calculated $\alpha_{\text {eff }} / n_{0}$ in the mixtures, we note relatively good agreement in the ionizationdominated region, except at the highest $E / n_{0}$, but some rather severe discrepancies in the attachment-dominated region. In the electronegative region, there were three separate adjustments made to the available cross sections, as we developed the database in I, that now appear inconsistent with the mixture measurements. The first of these occurs in the low field/energy region where a different rate of decrease in the coefficients is now observed between our pure and mixture measurements. The behavior of the pure THF $\alpha_{\text {eff }} / n_{0}$ was originally addressed by the low-energy extrapolation of the attachment cross section in our previous study. ${ }^{2}$ The second discrepancy between our calculated and experimental $\alpha_{\text {eff }} / n_{0}$ is in the "resonance" region just before the transition to ionizationdominated transport. Here, the previous large adjustment ${ }^{2}$ to the attachment cross section around $2.5 \mathrm{eV}$ now appears inconsistent with our new mixture measurements. Finally, we note that the transition from attachment- to ionization-dominated transport occurs at a (slightly) lower reduced electric field in our calculations compared to our measurements. This effect was addressed in our previous study ${ }^{2}$ by adjusting the threshold energy of the higher-threshold neutral dissociation channel.

The $E / n_{0}$ regions of the measured mixture transport coefficients that are not well captured by our calculated values are the same as those we addressed by the modifications in our previous study with pure THF. ${ }^{2}$ The disagreement we observe here suggests that those earlier adjustments to the cross sections have not resulted in a sufficiently accurate set to be consistent with the present mixture measurements. This observation emphasizes the need for mixture measurements in using the swarm inversion technique to derive a self-consistent set of scattering cross sections for a given system.

\section{Modifications to the THF cross section set}

The sensitivity of transport coefficients to variations within the error bars and extrapolations, of each of the individual cross sections, for electron impact with THF was discussed in I and will be used as a guide for the modifications required here as we attempt to reproduce the experimental mixture results. As in our previous study, ${ }^{2}$ the cross sections we consider for modification are those that are either not directly measurable, are difficult to measure, or are theoretically challenging. As noted above, the discrepancies between the measured and calculated coefficients shown in Figs. 1 and 2 occur at around the same features that were under- or overestimated by the "base set" of cross sections considered in I. Namely, the low-energy/field attachment and "resonance" regions of $\alpha_{\text {eff }} / n_{0}$, the change from attachment- to ionization-dominated transport in $\alpha_{\text {eff }} / n_{0}$, the low-energy/field region of the drift velocity, and the plateau region in the drift velocity which manifests as negative differential conductivity in the THF-Ar mixture measurements. As both the neutral dissociation and DEA cross sections can be challenging to measure and/or calculate, we have prioritized modifications to these cross sections over the others available in the respective energy regimes. The sensitivity of our mixture calculations to the errors in each of the other cross sections was tested, replicating the analysis in our previous study, ${ }^{2}$ but did not yield more preferable or more consistent results than the modifications proposed below, and so will not be detailed here explicitly.

a. Neutral dissociation. In our previous study I, we proposed the presence of two separate neutral dissociation cross sections to reconcile the underestimation of the drift velocity and to address the change to ionization-dominated transport in our calculations being at a lower $E / n_{0}$ compared with our experimental measurements in pure THF. Modifications to both of these cross sections are unfortunately now required to better reproduce the mixture measurements. These modifications are, however, consistent with the grand total cross section.

For the plateau/NDC region of the drift velocity present in both sets of mixture measurements, some changes to the lowerthreshold, neutral dissociation process cross section proposed in I were required. Changes to the threshold, shape, magnitude, and energy extent of this cross section were capable of better reproducing the experimental drift velocities. In the THF-Ar measurements, this was to better than $10 \%$ (with the exception of the 5\% THF95\% Ar mixture) and within $30 \%$ of the THF- $\mathrm{N}_{2}$ measurements (with the exception of the two mixtures with the lowest THF fractions). Our modified neutral dissociation process is characterized by a threshold reduced to $0.08 \mathrm{eV}$, a bimodal shape with the magnitude increased in the two separate energy regions to 2.5 times that in I (but still a 75\% reduction in the original Garland et al. ${ }^{1}$ remnant cross section) and an energy extent from the threshold to $3.5 \mathrm{eV}$, as labeled "current 1" in Fig. 5. The first peak in the cross section is necessary to increase the calculated drift velocity at the very start of the NDC region of the THF-Ar measurements, an underestimation particularly exposed by the low-THF-percentage mixtures. On the other hand, this effect is less important in the THF- $\mathrm{N}_{2}$ and high-THF-percentage THF-Ar measurements. At mean energies around $0.5 \mathrm{eV}$, corresponding to this underestimate of the drift velocity (by around $11 \%$ ), our pure argon calculations are in good agreement with the argon drift velocity data in the region, as shown in Fig. 11, reproducing Robertson et al. ${ }^{85}$ and Pack and Phelps ${ }^{86,87}$ to better than $5 \%$. Given this good agreement with the pure argon experimental measurements, we can with more confidence propose this change to the THF neutral dissociation cross section of I, despite its necessity only for the low-fraction THF admixtures with argon. In its absence, the energy exchange at these low energies changes and an alternative extrapolation toward low energies of the THF quasielastic cross section, discussed in Sec. IV B 3, would be required to better reproduce our present mixture measurements.

The second peak of the lower-energy threshold neutral dissociation process, and its increased energy range, over the cross section of I, reproduces the higher energy behavior in the plateau/NDC region, particularly the local minima of the THF-Ar NDC region. Embodied in Fig. 5 is a neutral dissociation cross section that suggests the presence of three neutral dissociation channels in THF. Such a scenario is entirely plausible.

Although the THF-Ar measurements expose this energy regime of the cross section set in more detail than the THF$\mathrm{N}_{2}$ and pure THF measurements, there probably still exists some nonuniqueness in the modifications we made to this neutral 
dissociation cross section that might reproduce the mixture measurements. For example, further modifications to the quasielastic cross section (separate to that discussed in the following paragraph) might be used as an alternative to the new lower-energy maximum in the proposed neutral dissociation cross section; however, these changes are not supported by the experimentally consistent quasielastic cross section (see Fig. 6).

In our previous study, ${ }^{2}$ we also discussed some of the possible nonuniqueness in the proposed cross section set. As an alternative to the then low-threshold neutral dissociation modifications, we commented that a decrease in the quasielastic momentum-transfer cross section, between 1 and $10 \mathrm{eV}$ and by up to $37 \%$, but outside the experimental error bars, produced similar drift velocities in good agreement with our pure THF measurements. To test the cross section set of I for self-consistency with the mixture measurements, this same quasielastic momentum-transfer modification was used to recalculate the drift velocities for all mixture compositions with both argon and $\mathrm{N}_{2}$. Significantly poorer agreement is now obtained than when using the modified low-threshold neutral dissociation cross section of Fig. 5 as the qualitative behavior in the NDC region of the THF-Ar mixtures could not be reproduced. This result supports our current proposed modifications to the neutral dissociation cross section, a process that is not yet able to be directly measured.

The plateau region of the drift velocity measurements, for the $1 \%$ and $2 \%$ THF additions to $\mathrm{N}_{2}$, however, remains overestimated by our calculations using the modified neutral dissociation cross section. From our sensitivity analysis, it is known that the drift velocity in this region can be influenced by the low-threshold neutral dissociation and quasielastic momentum-transfer cross sections. However, reproducing the experimental drift velocities of these two fractions requires modifications, to both of these cross sections, which are incompatible with the results for all the other mixture fractionsthe lower-threshold neutral dissociation process would need to be decreased substantially in magnitude, or removed entirely, accompanied by a decrease in the quasielastic momentum-transfer cross section. We return to this apparent paradox, for the $1 \%$ and $2 \%$ admixtures of THF in $\mathrm{N}_{2}$, later. At this time, we simply note that it is the higher percentage THF fraction measurements $(\geq 5 \%)$ that were favored for our further analysis of the cross section set modifications.

Using the THF cross section set developed previously in I, the effective Townsend ionization coefficient calculated for the THF-Ar mixtures changes from attachment- to ionization-dominated transport at an earlier $E / n_{0}$ than our measurements. This is similar to the behavior of our pure THF calculations using our "base set" in I (see Fig. 5 in I). Decreasing the threshold of the higher neutral dissociation process to $7 \mathrm{eV}$, and shifting the entire cross section down by $3 \mathrm{eV}$ (from the $10 \mathrm{eV}$ threshold proposed in I), is now necessary to better reproduce the experimental mixture measurements. Other modifications to the magnitude and threshold of the neutral dissociation cross section can also assist us to reasonably reproduce the mixture results for this coefficient. The reduction in the third peak (labeled "current 2" in Fig. 5) threshold to $7 \mathrm{eV}$ causes a modification of the energy and momentum transfer rates, resulting in a slight increase in the drift velocity at the very end of the plateau region. This has the effect of moving our simulated drift velocities a little away from experimental measurements; however, this adjustment was favored for its relatively smooth appearance compared to more complicated alternatives. In this transition region from electronegative transport, the pure THF $\alpha_{\text {eff }} / n_{0}$ calculated using this cross section still reasonably reproduces the experimental data (as shown in Fig. 7), highlighting the nonuniqueness possible in a cross section set developed when only one set of experimental transport measurements is available to probe that set, such as in I.

b. Dissociative electron attachment. Three main energy regions of the original attachment/DEA cross section (hereafter referred to as the DEA cross section) require modifications from that proposed in I to better reproduce the experimental mixture measurements.

The first adjustment addresses the low-field attachmentdominated region of $\alpha_{\text {eff }} / n_{0}$, which shows a smaller rate of change
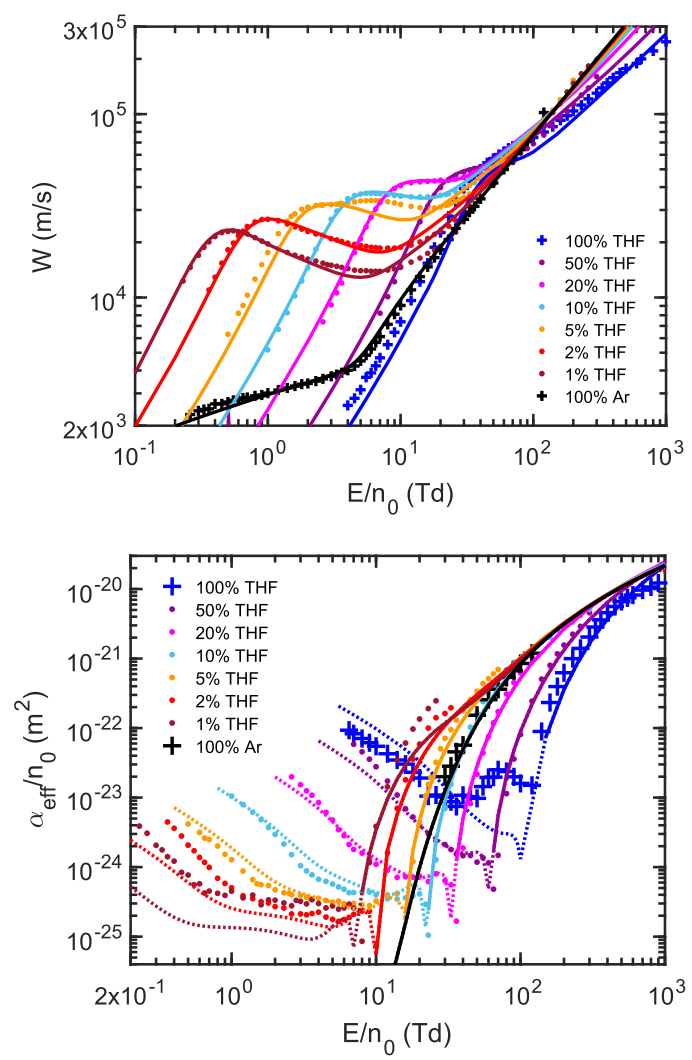

FIG. 7. (Upper) The electron drift velocity and (lower) the effective Townsend ionization coefficient in THF-Ar mixtures as a function of the reduced electric field $E / n_{0}$. The symbols correspond to our measurements, while the lines represent our calculated values using the modified cross section set proposed in Sec. IV B 3. The broken lines correspond to the electronegative region of the effective Townsend ionization coefficient with the change to ionization-dominated transport denoted by the solid lines. The behavior in this region is generally well captured by our calculations for the mixture measurements although the absolute difference can be large, as shown in the middle panel of Fig. 9. Note that the values for the curves with $1 \%-5 \%$ THF are to be taken as only trends (see Sec. II). 
in the attachment rate in mixtures with argon than in the pure THF measurements. The general trend of our calculations, using cross section set $\mathrm{I}$, is an underestimate of the experimental measurements in the low-energy/field region, as shown in Fig. 1. This region is dominated by the extrapolated part of the DEA cross section, at energies below where experimental results are available. To address the underestimation, the extrapolation of the cross section proposed in I must be modified to now reduce the DEA magnitude near the region of the lowest experimental data, with a slight magnitude increase at energies just below this, as shown in Fig. 4. The mixtures with higher fractions of THF show a different rate of change in the electronegative region, as compared to the pure THF and the mixtures with lower proportions of THF, so our adjustments to the DEA cross section were selected to minimize the deviations between simulation and measurement for all the mixture measurements simultaneously.

The "resonance" feature present in the electronegative region of the pure THF $\alpha_{\text {eff }} / n_{0}$, i.e., measurements from 70 to $120 \mathrm{Td}$, is significantly suppressed in the argon mixture measurements, with the signature appearing to decrease with increasing fractions of THF. To reproduce the mixture measurements, the adjustment made in I to the DEA cross section at around $2.5 \mathrm{eV}$, to reproduce the pure THF measurements, is now not compatible. This lends weight to our speculation in I for the possibility that a small impurity was present in our pure THF measurement. This assertion follows as the large increase in the DEA cross section around $2.5 \mathrm{eV}$, required to reproduce the pure THF Townsend coefficient, was much larger than the associated experimental error bars, as shown in Fig. $4 .{ }^{88}$

Finally, to reproduce the vestige of the "resonance" feature, present as a small-range slight magnitude increase in the electronegative region of the mixture measurements, just before $\alpha_{\text {eff }} / n_{0}$ changes to the ionization-dominated region, and to reproduce the (absolute) minimum in this coefficient, requires an increase in the magnitude of the DEA cross section above $5 \mathrm{eV}$ by a factor of two and above $7.4 \mathrm{eV}$ by a factor of six times over the magnitude of the measurements of Janećková et al. ${ }^{75}$ used in the modified cross section proposed in I. We note that DEA measurements, in terms of setting the absolute scale, are notoriously difficult, particularly when this absolute magnitude is very small (note the y-axis of Fig. 4). Thus, adjustments to its magnitude, such as we propose here, are by no means implausible.

The larger $\alpha_{\text {eff }} / n_{0}$ measured for the $1 \%$ THF-99\% Ar mixture fraction, from 1 to $8 \mathrm{Td}$, compared to the $2 \%$ THF- $98 \%$ Ar mixture, can be reproduced by including an increase in the DEA cross section at around $2.5 \mathrm{eV}$, similar to but smaller than that previously proposed in I. Nonetheless, this inconsistency for the necessity of this increase in the DEA cross section magnitude leads us to also consider the possibility of impurities in our 1\% THF-99\% Ar mixture or the presence of Penning ionization.

c. Quasielastic scattering. Comparing our measured and calculated drift velocities, at values of $E / n_{0}$ below the plateau/NDC region, we observe that using the cross section set developed in I generally results in an overestimation by the simulation, except for the three lowest THF admixture fractions with $\mathrm{N}_{2}$. In this energy regime, from a knowledge of our sensitivity analysis, ${ }^{2}$ the quasielastic momentum-transfer cross section, below where experimental data are available, and low-threshold neutral dissociation pathways are candidates for adjustment. Changes to the low-threshold neutral dissociation cross section can produce a decrease in the magnitude of the calculated drift velocity, however, to replicate our mixture measurements requires a change in both the magnitude and rate of increase in our calculated drift velocity. This can be achieved by an adjustment to the original low-energy quasielastic momentum-transfer extrapolation, ${ }^{2}$ at energies below the lowest experimental datum of Gauf et al. ${ }^{81}$ at $0.75 \mathrm{eV}$ (see Fig. 3).

The amount the drift velocity is overestimated by our calculation changes for each mixture fraction, in both gas mixtures, and this results in some difficulty in finding a single modification to the cross section set to reproduce all the mixture fractions simultaneously. Generally, for the THF-Ar mixtures, the drift velocities are overestimated by our simulation, while for the THF- $\mathrm{N}_{2}$ mixtures, they are underestimated. To minimize the discrepancies between our measured and calculated drift velocities, the extrapolation of the quasielastic momentum-transfer cross section below $0.2 \mathrm{eV}$ was modified to have a magnitude of $175 \times 10^{-20} \mathrm{~m}^{2}$ at $0.1 \mathrm{eV}$.

We find here that any effect in resolving the quasielastic cross section into separate elastic and rotational components has not been exposed by our present mixture measurements. Indeed the rate of increase in the drift velocity, at $E / n_{0}$ lower than our present measurements, is only where we see changes due to inclusion of an explicit rotational excitation cross section. This is where the mean energy of the electron swarm is approaching thermal energies.

For the modifications proposed in this study, we always aim to conserve the grand total cross section (TCS) of the Madrid group, ${ }^{24}$ as in our previous study. ${ }^{2}$ To preserve this TCS, modifications to the quasielastic integral cross section (ICS) are required in order to compensate for our previously discussed adjustments to the DEA and neutral dissociation cross sections. Since elastic collisions (or quasielastic here) are sampled through the (quasi)elastic momentum-transfer cross section, for a Boltzmann equation solution, modifications to the (quasi)elastic ICS are not explicitly tested. Nonetheless the necessary modification to the quasielastic ICS must remain consistent with the available experimental measurements and theoretical estimates, and our proposed new cross section satisfies this condition, as is shown in Fig. 6.

d. Transport coefficients simulated using the new set. The transport coefficients calculated using the proposed modifications to the cross section set of I are shown in Figs. 7 and 8. Given the overlap of the data, in Fig. 9, we display the percentage differences between our experimental measurements and calculated transport coefficients. Owing to the adjustments to the cross section set needed to better reproduce the mixture measurements, the differences between our pure THF calculated and measured coefficients have increased above what we found using set I below $200 \mathrm{Td}$. For the drift velocity, this difference is now up to $30 \%$, where our measurements are generally underestimated by the simulation with the cross section changes proposed here. Above 200 $\mathrm{Td}$, however, our present calculations remain in quite good agreement with those of our previous study, with the error between theory and measurements reducing to $\leq 10 \%$. The pure THF effective 


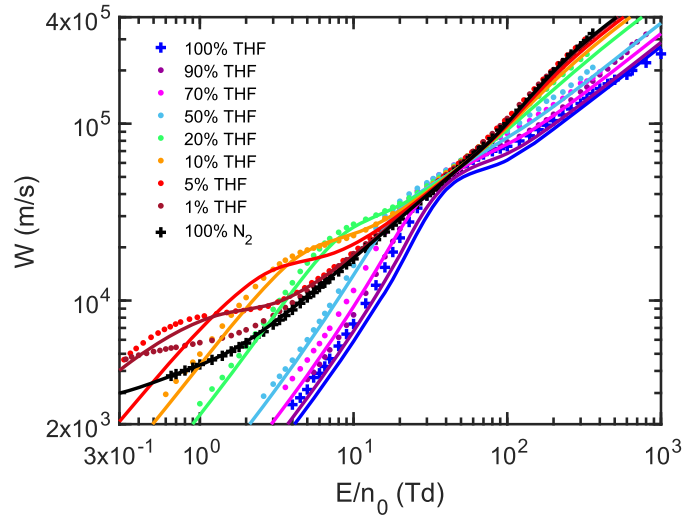

FIG. 8. The electron drift velocity in THF- $\mathrm{N}_{2}$ mixtures, as a function of the reduced electric field $E / n_{0}$. The symbols correspond to our measurements, while the lines represent our calculated values using the modified cross section set proposed in Sec. IV B 3 e. The coincidence of the $5 \%$ THF- $95 \% \mathrm{~N}_{2}$ measurements with the $1 \%$ THF- $99 \% \mathrm{~N}_{2}$ calculated drift velocities is not erroneous and is discussed in paragraph Sec. IV B 3 e.

Townsend ionization coefficient can now differ by up to $100 \%$ in the electronegative attachment region due to the different behaviors exhibited in the mixture measurements. The change to ionizationdominated transport is now calculated at a slightly higher field than our experimental measurements. Similar to, but lower in magnitude than our previous calculations in I, in the electropositive region, our present calculations differ from the measurements by $\leq 50 \%$. In this energy regime, the ionization and higher threshold neutral dissociation cross sections dominate the collisional energy exchange. We were unable to find an adjustment to the neutral dissociation cross section to better reproduce the ionization coefficient, and given the good agreement between the experimental crossed-beam and calculated ionization cross sections, we are reluctant to postulate changes to this cross section in order to reduce the observed differences.

For our mixture measurements, we generally find reasonable agreement between the calculated and measured drift velocities over the range of $E / n_{0}$ sampled, where we reproduce the experimental values to within $\pm 30 \%$, with the exception of the two lowest fractions of THF in $\mathrm{N}_{2}$ mixtures which are discussed in Subsection IV B 3 e. The discrepancies are greatest at the lower $E / n_{0}$, where the choice of the extrapolation of the quasielastic momentumtransfer cross section can minimize errors in each mixture fraction individually. However, we were unable to find an adjustment to reproduce all the mixture fractions simultaneously. In mixtures with argon, at reduced electric fields above $200 \mathrm{Td}$, we observe a larger underestimation in the simulated drift velocities due in part to the underestimation by our simulation in the pure argon drift velocity profile.

Comparisons of $\alpha_{\text {eff }} / n_{0}$ in the argon mixtures with our experimental values are less favorable than with the drift velocities. In this case, there are discrepancies up to $\pm 100 \%$, as shown in the middle panel of Fig. 9. Exceptions to this occur around the minimum absolute value of $\alpha_{\text {eff }} / n_{0}$ for the pure and mixture measurements that are not entirely captured by our calculations. A large source of those
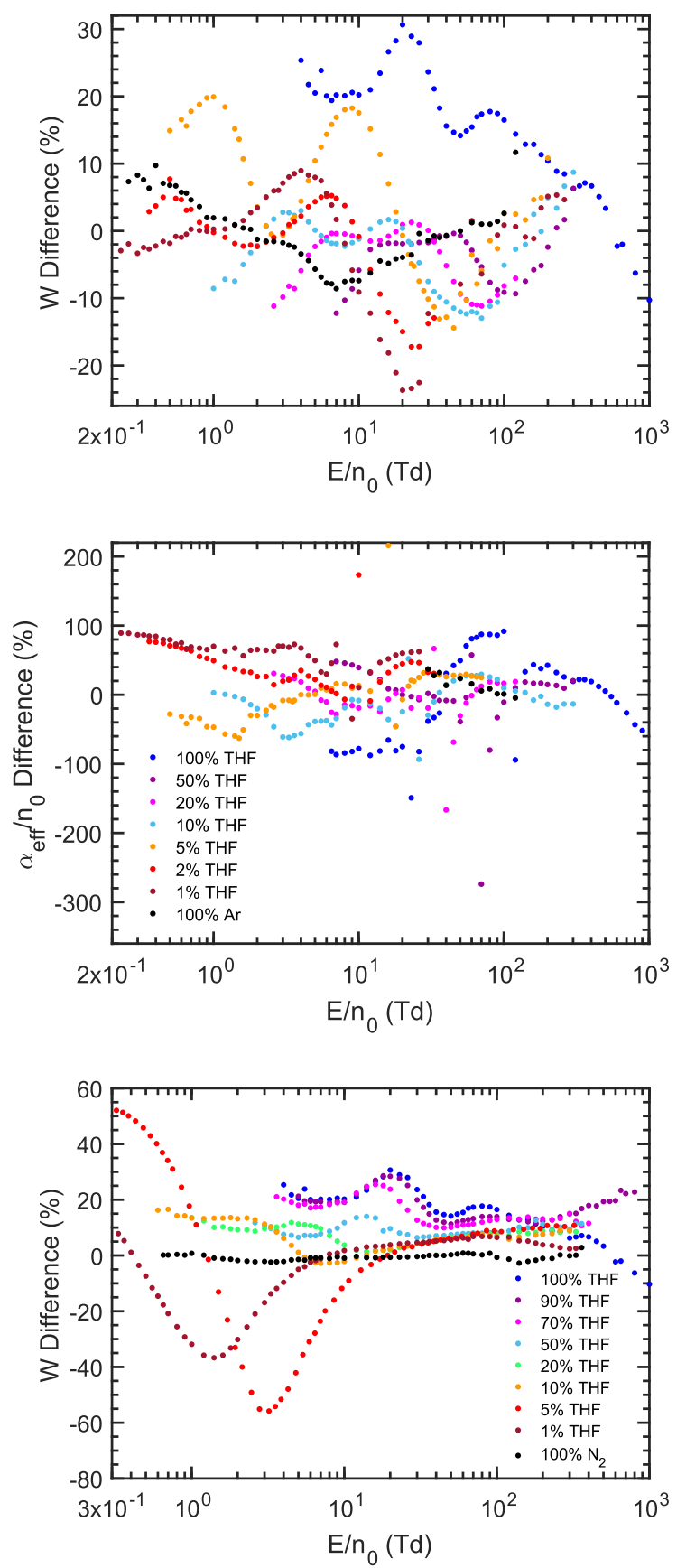

FIG. 9. The \% difference between our calculated and measured transport coefficients for an electron swarm in admixtures of THF with argon and $\mathrm{N}_{2}$ as a function of the reduced electric field. The transport coefficients are calculated using the modifications to the cross section set proposed in Sec. IV B 3. (Upper) The upper panel shows the percentage difference between the measured and calculated drift velocity for admixtures with argon, with the legend in the middle panel corresponding to the same colors/mixture compositions. (Middle) The middle panel shows the percentage difference between the calculated and measured effective Townsend ionization coefficients in mixtures of THF-Ar. (Lower) The lower panel shows the percentage difference between the calculated and measured drift velocities in mixtures of THF- $\mathrm{N}_{2}$. 
differences is in the extrapolated value of the DEA cross section to lower energies (below the available experimental values), where our simulations show that the particular cross section value chosen can minimize these errors for one mixture fraction at a time, but not all mixture fractions simultaneously. The change from electronegative to electropositive transport recorded in our measurements is generally well captured by our calculations, occurring at the correct field for the three higher and lowest of the THF fractions, and at a slightly lower $E / n_{0}$ for the $2 \%-10 \%$ THF admixture profiles.

Referring to the lower panel of Figs. 7 and 9, the $\alpha_{\text {eff }} / n_{0}$ values for the THF-Ar mixtures containing relatively small amounts of THF, from $1 \%$ to $5 \%$, we note that the experimental curves deviate systematically from the calculated ones in the electronegative region. This is very likely to be due to a second source of ionization arising from Penning effects. This happens because the first metastable level of $\mathrm{Ar}$ at around $11 \mathrm{eV}$ is slightly higher than the
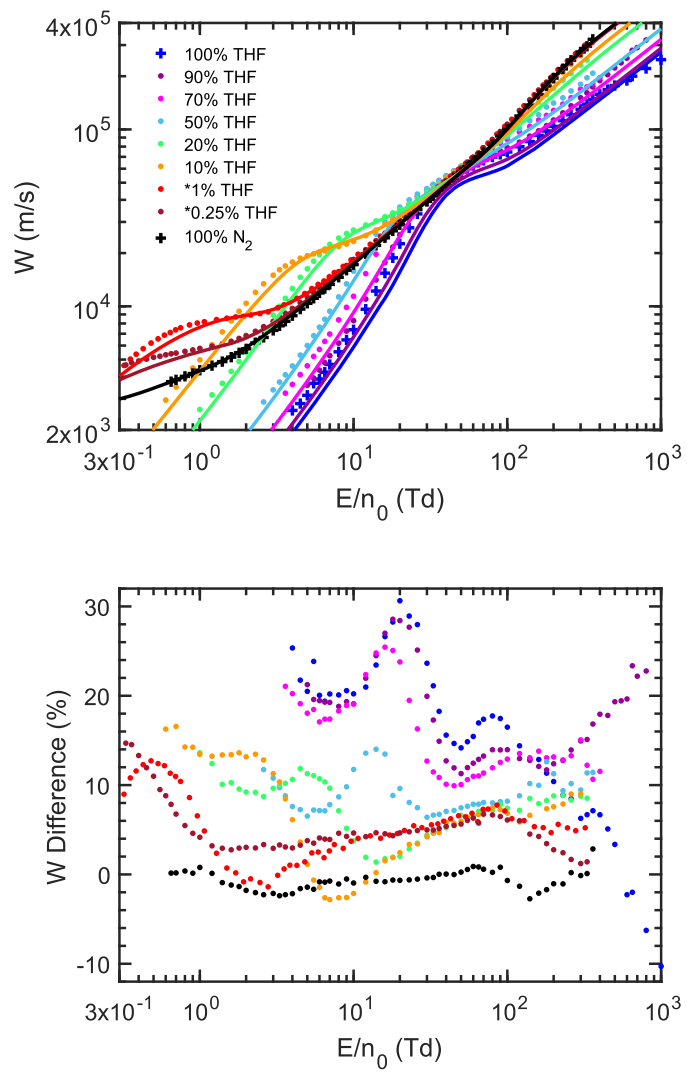

FIG. 10. (Upper) Comparison of the experimental and calculated drift velocities for electrons in mixtures of THF and $\mathrm{N}_{2}$, as a function of $E / n_{0}$, when the mixture composition of the two lowest THF admixture calculations are adjusted from $5 \%$ and $1 \%$ THF, to $1 \%$ and $0.25 \%$ THF, respectively. (Lower) The differences between the calculated and measured drift velocities for mixtures of THF in $\mathrm{N}_{2}$, for mixture compositions adjusted from $5 \%$ and $1 \%$ THF in $\mathrm{N}_{2}$, to $1 \%$ and $0.25 \%$ THF in $\mathrm{N}_{2}$. The legend in the upper panel also corresponds to the lower panel, where the asterisk denotes the mixture compositions that have been adjusted for the corresponding simulation. ionization energy of the THF molecule of $9.40 \pm 0.02 \mathrm{eV} .{ }^{89}$ Using the same pulsed Townsend technique, this effect has been found in $\mathrm{C}_{2} \mathrm{~F}_{4}$-Ar mixtures, ${ }^{90}$ while no trace of it was found in $\mathrm{C}_{2} \mathrm{~F}_{4}-\mathrm{Xe}$ mixtures since the first metastable level of Xe lies below the ionization energy of $\mathrm{C}_{2} \mathrm{~F}_{4}{ }^{91}$ At the higher THF-content mixtures, electron impact ionization predominates over any possible Penning ionization.

e. Mixture fractions. Measurements of the drift velocities for the two lowest fractions of THF mixtures with $\mathrm{N}_{2}$ are much lower in magnitude than our calculated values at the same mixture compositions. These differences were not able to be resolved with modifications to the cross section set and for our results to remain largely consistent with each of the other mixture measurements, including those in THF-Ar. Given the disparity for the lowest THF fractions in $\mathrm{N}_{2}$, we have also assessed the variation in the drift velocity within the error bars associated with the gas mixture composition, $\pm 0.3 \%$, but were unable to resolve the differences. However, given the very near agreement between the 5\% THF-95\% $\mathrm{N}_{2}$ measurements and the $1 \%$ THF- $99 \% \mathrm{~N}_{2}$ calculations in Fig. 8 , we have investigated if an alternative mixture ratio could reproduce the measured 1\% THF-99\% $\mathrm{N}_{2}$ mixture behavior. Figure 10 shows the drift velocity calculated for a $0.25 \%$ THF- $99.75 \% \mathrm{~N}_{2}$ mixture composition, and the differences between our measurements and calculations if the actual mixture compositions of the two lowest THF admixtures to $\mathrm{N}_{2}$ measurements were taken to be $1 \%$ THF- $99 \% \mathrm{~N}_{2}$ and $0.25 \%$ THF- $99.75 \% \mathrm{~N}_{2}$. Using this adjusted fractional composition, the calculated drift velocities reproduce the measurements in both cases with significantly reduced errors, and in line with the differences we find for the higher THF fractional mixtures. This speculation suggests the possibility, given that THF is a rather "sticky" substance, that at the lowest THF admixtures in $\mathrm{N}_{2}$, the actual mixture composition that the electron swarm traverses through might be a little different from the bulk composition we measure.

\section{FINAL REMARKS}

This study presents the first measurements of electron swarm transport coefficients in mixtures of THF with $\mathrm{Ar}$ and $\mathrm{N}_{2}$. THF is a biologically relevant molecule that is one of the most comprehensively studied (second only to water) in terms of its scattering cross sections and swarm measurements (detailed for pure THF in our previous study ${ }^{2}$ ). Using the pulsed Townsend technique, the electron drift velocity and the effective Townsend ionization coefficient have been measured over a range of reduced electric fields $0.23 \leq E / n_{0} \leq 800 \mathrm{Td}$ for various mixture ratios of THF with argon and $\mathrm{N}_{2}$. Using a multiterm kinetic theory solution, these transport coefficients were also simulated using self-consistent and often employed cross sections for argon and $\mathrm{N}_{2}$ (see the Appendix), and the set of THF scattering cross sections developed in our previous swarm analysis. ${ }^{2}$ In this earlier study, the best available compilation of a complete THF scattering cross section set, developed in the work of Garland et al., ${ }^{1}$ was adjusted to reproduce the pure THF swarm measurements. This study represents an important extension to that earlier work, here using mixture measurements as a further test of the accuracy and completeness of our previously proposed set. We observed some quantitative differences between the simulated 
and experimental transport coefficients in the mixture measurements and the pure THF measurements, which required adjustments to the cross section set derived in I to try and better reproduce the measured $E / n_{0}$ dependence of the drift velocities and the effective Townsend ionization coefficients.

To best reproduce the mixture measurements, here, we proposed adjustments to the cross sections that are generally difficult to measure or calculate, namely, the DEA and neutral dissociation cross sections, as well as to the low-energy extrapolation of the quasielastic cross section. Although the present modifications have somewhat worsened the reproducibility of the pure THF measurements compared to those in I, in some regions of $E / n_{0}$, the additional mixture measurements have confirmed the rationale behind our previous cross section adjustments. While some of the nonuniqueness known to exist in the modified cross section set proposed in I has now been resolved, the additional experimental data of this investigation has resulted in a greater uncertainty in the validity of the low-energy extrapolation of the quasielastic and DEA cross sections. The present mixture measurements have also been unable to expose regions of the cross section set that would allow discrimination between the elastic and rotational contributions to the quasielastic cross section.

We remain cautious that the modifications proposed here have resulted in the most accurate set of THF scattering cross sections although we are confident we have made some important progress toward this goal. This investigation has, however, highlighted the necessity of the full swarm inversion technique, with multiple sets of experimental data, for assessing cross sections which are difficult to measure, e.g., for the neutral dissociation process and for the dissociative electron attachment magnitude. Our future studies will focus on resolving some of the uncertainties that still remain in the gaseous THF cross section set, by exposing different energy regimes, possibly through the use of different temperatures or under crossed electric and magnetic fields. We also aim to extend the gas-phase cross sections to the condensed or liquid phase, as detailed in the work of White and Robson. ${ }^{61,92}$ This and our future studies represent a necessary step in assessing the accuracy and completeness of scattering cross sections for biologically relevant molecules, with the eventual aim of modeling ionizing radiation treatments and damage due to secondary electrons in biological tissue. $^{93,94}$

\section{SUPPLEMENTARY MATERIAL}

See the supplementary material for the experimental measurements of the drift velocity and the effective Townsend ionization coefficient in mixtures of THF with argon and $\mathrm{N}_{2}$.

\section{ACKNOWLEDGMENTS}

The authors would like to thank the Australian Research Council through its Discovery Program (No. DP180101655) for financial support. The experimental work was supported by UNAM-PAPIIT IN 108417 and Conacyt Project No. 240073. The technical assistance of A. Bustos and G. Bustos is greatly acknowledged.

\section{APPENDIX: ARGON AND $\mathbf{N}_{2}$}

In this appendix and in the supplementary tables, we report our experimental measurements of the drift velocity and the Townsend ionization coefficient in pure argon and pure $\mathrm{N}_{2}$. The particular cross section sets employed in this investigation for argon and $\mathrm{N}_{2}$ are described and used to calculate the drift velocity and $\alpha_{T} / n_{0}$ (in the absence of any attachment processes) in both of the pure gases. Our present calculations are compared with our current experimental measurements and some of the other available experimental data. We note that the pure $\mathrm{N}_{2}$ transport coefficients, both our measured and calculated, were recently reported in the work of Casey et al. ${ }^{67}$ and are summarized in this appendix for completeness.

\section{Pulsed Townsend measurements of the drift velocity and the effective Townsend ionization coefficient}

\section{a. Argon}

Our experimental measurements of the drift velocity and $\alpha_{T} / n_{0}$ of a swarm of electrons in pure argon, as a function of the reduced electric field $E / n_{0}$, are presented in Fig. 11. These measurements are compared with the other swarm data available in the literature, where we find agreement is generally better than $10 \%$, except in the high-field region where the difference between our present measurements and those of Hernández-Ávila et al. ${ }^{71,95}$ increases up to $20 \%$. The difference between the various experimental measurements of $\alpha_{T} / n_{0}$ in argon is larger than those of the drift velocity, but generally our present experimental measurements lie comfortably within the majority of the available data.

\section{b. Nitrogen}

Swarms of electrons in $\mathrm{N}_{2}$ have also been extensively studied, ${ }^{105}$ and in Fig. 12, we compare our measurements of the drift velocity and the Townsend ionization coefficient in pure $\mathrm{N}_{2}$, with some of the other available experimental results. Our measured drift velocity generally lies below the other experimental measurements, by at most $10 \%$. Measurements of the effective Townsend ionization coefficient in $\mathrm{N}_{2}$ also have a quite large spread in values, and our measurements are generally below but within $50 \%$ of the other experimental results as shown in the lower panel of Fig. 12.

\section{Cross section sets}

\section{a. Argon}

The set of argon cross sections utilized here are from the work of Biagi from the MAGBOLTZ v8.97 database tabulated on LXCat. ${ }^{131}$ This set consists of an elastic cross section, an ionization cross section, and 44 individual electronic-state excitations, the derivation and assessment of which have been described elsewhere. ${ }^{132-134}$ Given the low field and mean energy range of the calculations in this study, we also include the low-energy extrapolation of the elastic cross section using modified effective range theory as is utilized in MAGBOLTZ. ${ }^{132}$ Our present simulations using this set are discussed in the following subsection and compared to our current measurements and some of the other available swarm data in 

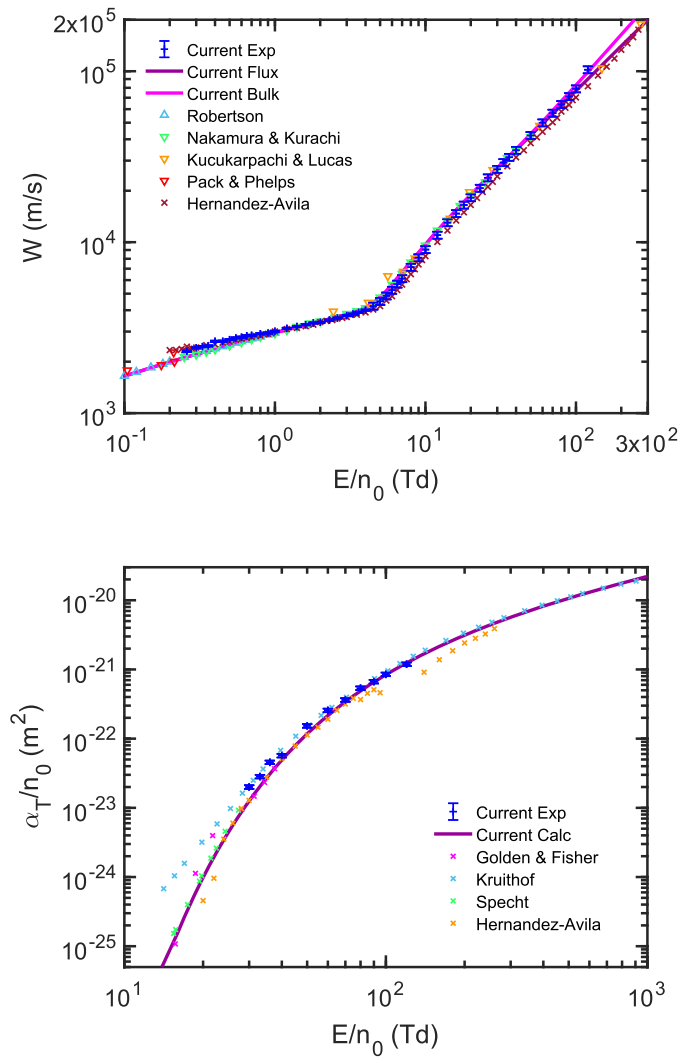

FIG. 11. (Upper) The experimental drift velocity for an electron swarm in Ar, as a function of reduced electric field, compared with our calculated values and the measurements of Hernández-Ávila et al., ${ }^{71,95}$ Robertson (with uncertainty reported at $3 \%$ ), ${ }^{85}$ Pack and Phelps at $300 \mathrm{~K}^{86,87}$ (the presence of molecular impurities in this experiment could result in an uncertainty of up to $25 \%$, as assessed in Ref. 85), Kucukarpachi and Lucas, ${ }^{96,97}$ and Nakamura and Kurachi ${ }^{98}$ (with error bars reported at $2 \%$ ). (Lower) Comparison of our measured and calculated Townsend ionization coefficient with the experimental measurements of Golden and Fisher, ${ }^{99,100}$ Kruithof, ${ }^{101,102}$ Specht, ${ }^{103,104}$ and Hernández-Ávila et al.$^{71,95}$ The present calculations are performed using the cross section set detailed in subsection 2 a of the Appendix.

Fig. 11. We note that a differential elastic cross section can be used to derive higher-order partial cross sections, but as investigated in the work of Boyle et al., ${ }^{69}$ the inclusion of higher-orders results in a less than $1 \%$ change (and usually less than $0.1 \%$ ) in the transport coefficients for argon.

It should also be briefly noted that while the argon Biagi cross section database was able to provide reasonable self-consistency between our simulated transport coefficients, and those from measurements, the cross sections compiled are not necessarily in agreement with those from crossed-beam measurements or scattering theory. Thus, the potential for nonuniqueness arises here.

\section{b. Nitrogen}

The set of cross sections for $\mathrm{N}_{2}$ used in the following work were also taken from Biagi's MAGBOLTZ v8.97 database tabulated on
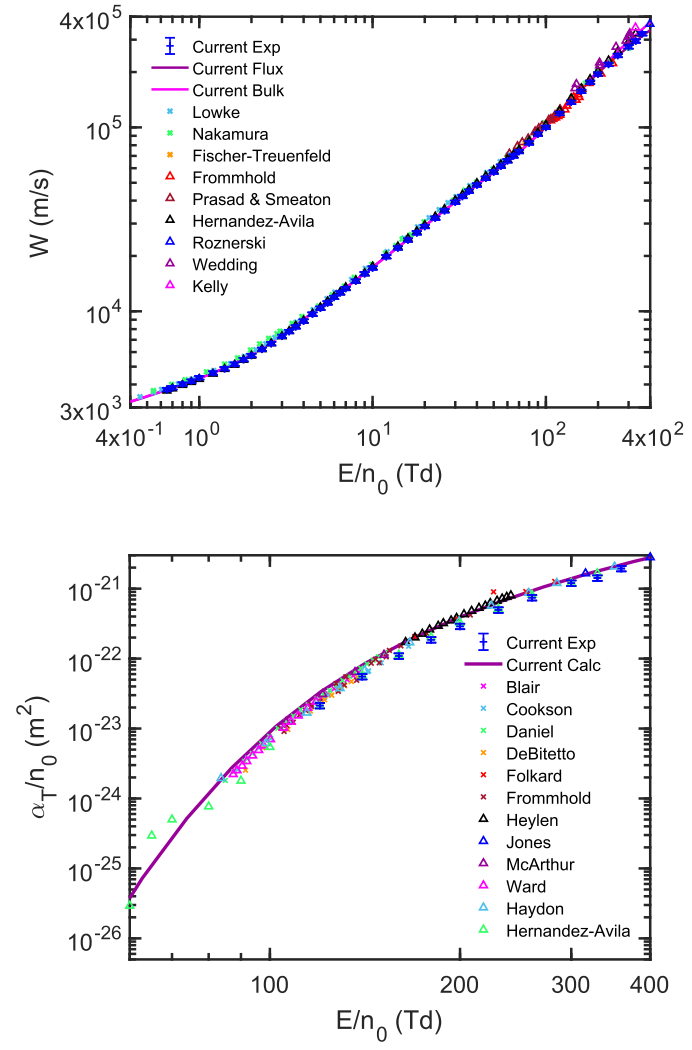

FIG. 12. (Upper) Comparison of the present experimental and simulated drift velocity for an electron swarm in pure $\mathrm{N}_{2}$ at $300 \mathrm{~K}$ as a function of the reduced electric field. These are also compared with the earlier measurements of Lowke at $293 \mathrm{~K},{ }^{106}$ Nakamura at $293 \mathrm{~K},{ }^{107}$ Fischer-Treuenfeld, ${ }^{108,109}$ Frommhold, ${ }^{11}$ Prasad and Smeaton, ${ }^{112,113}$ Hernández-Ávila et al., ${ }^{71}$ Wedding et al., ${ }^{114}$ Roznerski, ${ }^{115,116}$ and Kelly ${ }^{117}$ (digitized from Campbell et al. ${ }^{118}$ ). (Lower) Comparison of the Townsend ionization coefficient, measured and calculated in the present study, and compared with the experimental measurements of Bagnal and Haydon,

Blair, ${ }^{120}$ Cookson et al., ${ }^{121}$ Daniel and Harris, ${ }^{122}$ DeBitetto and Fisher, ${ }^{123}$ Dutton et al., ${ }^{124}$ Folkard and Haydon, ${ }^{125}$ Frommhold, ${ }^{111}$ Heylen, ${ }^{126}$ Jones, ${ }^{127}$ McArthur and Tedford, ${ }^{128}$ Ward, ${ }^{129}$ Haydon and Williams, ${ }^{130}$ Hernández-Ávila et al., Kelly ${ }^{117}$ (digitized from Campbell et al. ${ }^{118}$ ), and Wedding et al..$^{114}$ The present calculations are performed using the cross section set detailed in subsection $2 \mathrm{~b}$ of the Appendix.

LXCat. ${ }^{131}$ The tabulated set contains an elastic momentum-transfer, 15 individual vibrational states, 29 individual electronic states, and a single ionization cross section. The elastic momentum-transfer cross section tabulated on LXCat is more sparse at lower energies than the original source, so the data of Itikawa ${ }^{135}$ is included below $0.07 \mathrm{eV}$.

At the time of this study, no rotational cross sections had been included in the Biagi databases on LXCat, and although other databases do include a description of rotational collisions, our preference here (and in Ref. 67) is to extract the rotational cross sections detailed in Biagi's MAGBOLTZ source code, which include scalings compatible with the author's resonance region and elastic cross section. Taken from the Gas16 tabulation, rotational 
collisions for $j \rightarrow j+2$ transitions are included for no less than the 40 lowest transitions, calculated using the Gerjuoy and Stein treatment, ${ }^{136,137}$ with an enhancement in the resonance region between 1.2 and $5.3 \mathrm{eV}$, and scaling of the cross sections above $5 \mathrm{eV}$ to fall at the same rate as the elastic momentum-transfer cross section. Our calculations using this cross section set are shown in Fig. 12 and compared with our current experimental measurements, and those of other authors, in the following section.

In addition to what we have noted for argon, while the $\mathrm{N}_{2}$ Biagi cross section database was also able to produce reasonable self-consistency between our simulated transport coefficients, at the two-term level for $\alpha_{T} / n_{0}$ as discussed below, and those from measurements, the cross sections are not necessarily in agreement with those from crossed-beam measurements or scattering theory. Thus, the potential for nonuniqueness also exists here.

\section{Comparison of transport coefficients}

In this section, we assess the differences between our calculated transport coefficients in the pure $\mathrm{Ar}$ and $\mathrm{N}_{2}$ gases and the corresponding available experimental measurements. We further consider the differences between the different drift velocities that can be extracted from the analysis of the different experimental methods.

\section{a. Argon}

The three different definitions of the drift velocity considered herein vary most at the highest $E / n_{0}$, where ionization processes have the greatest effect on the electron swarm. For argon, the bulk drift velocity is up to $37 \%$ greater than the flux drift velocity at 1000 $\mathrm{Td}$, where the steady-state Townsend drift velocity is up to $14 \%$ lower than the flux drift velocity. Now comparing our calculated flux drift velocity with the experimental measurements, we find agreement generally to within $10 \%$. At the highest reduced electric fields, however, our experimental measurements are up to $12 \%$ above the calculated flux drift velocity, which is greater than the experimental error bars of $\pm 1 \%$ for $E / n_{0} \leq 4 \mathrm{Td}$ and $\pm 4.7 \%$ above this. When comparing with our calculated bulk drift velocity, however, these differences reduce to below $3 \%$, within the experimental uncertainty. Conversely, our calculated flux drift velocity compares well with the earlier measurements of Hernández-Ávila et al. ${ }^{71,95}$ (with a reported experimental accuracy of $2 \%$ ), with a difference of less than $7 \%$, that increases to $20 \%$ when comparing to our calculated bulk drift velocity. Below $1 \mathrm{Td}$, our calculated drift velocity underestimates both our present measurements and those of Hernández-Ávila et al. by up to $10 \%$ and $18 \%$, respectively.

There is a large volume of experimental measurements for the Townsend ionization coefficient in argon, so we only consider the measurements selected in the review by Pitchford et al. ${ }^{134}$ Similar to the situation when we compare the simulation results to our current experimental measurements, generally our calculated values are to within $50 \%$ of the other experimental values. However, this difference increases to over $100 \%$ at the lower $E / n_{0}$ in the measurements of Golden and Fisher, ${ }^{99,100}$ Kruithof $^{101,102}$ (likely to contain a Penning component at low fields ${ }^{134}$ ), and Specht. ${ }^{103,104}$ The difference between our current experimental measurements and our calculated Townsend ionization coefficient is typically within $30 \%$, which is still larger than the error bars of $\pm 8 \%$ on our experimental measurements.
The sensitivity of the transport coefficients to measurement temperature variations, to within the experimental error bars, is greatest at the lowest $E / n_{0}$ considered. Here, the calculated transport coefficients can vary by up to $44 \%$ and $20 \%$, for the flux drift velocity and the mean energy, respectively, when simulated at $293 \pm 0.5 \mathrm{~K}$. That difference decreases with increasing $E / n_{0}$, to $<1 \%$ difference above $33 \mathrm{Td}$ and $14 \mathrm{Td}$ for the two coefficients, respectively. The Townsend ionization coefficient is less sensitive to temperature, varying by only up to $\pm 0.3 \%$. The effect of the particular energy sharing between the two postionization electrons is also considered. Throughout this study, we have taken the energy sharing fraction to be equal between the indistinguishable scattered and ejected electrons, but we also consider the effect of the extreme case of $1 \%-$ $99 \%$ sharing between the two outgoing electrons and all-fractionsequiprobable (AFE) sharing. At $120 \mathrm{Td}$, the highest measured $E / n_{0}$, the variation of the transport coefficients compared to the $50 \%-50 \%$ energy-sharing case is less than $0.7 \%$ for the $1 \%-99 \%$ case and less than $0.5 \%$ for the AFE case in argon.

\section{b. Nitrogen}

Our calculated flux drift velocities for $\mathrm{N}_{2}$ are in good agreement with our current experimental measurements, compatible with the overall experimental uncertainty of $\pm 2.2 \%$, as shown in Fig. 12 . The calculated flux drift velocity $(W)$ is within $2.8 \%$ of our measurements, while the bulk drift velocity $\left(W_{B}\right)$ is within $8 \%$ and the steady-state Townsend drift velocity $\left(W_{S S T}\right)$ is up to $5 \%$ below our measurements. These differences increase with increasing $E / n_{0}$ as the influence of ionization increases. Below $200 \mathrm{Td}$, our measured flux drift velocity generally underestimates all the other available experimental measurements by between $7 \%$ and $20 \%$.

The agreement between our calculated and experimental Townsend ionization coefficient is not as favorable, with our simulated values overestimating our measurements by between $14 \%$ and $55 \%$, greater than the overall uncertainty in the measurements of $\sim \pm 9 \%$. That variance, however, decreases with increasing $E / n_{0}$. Compared to the other available measurements, our present calculations tend to overestimate the experiments below $150 \mathrm{Td}$ and underestimate them above this to generally less than $55 \%$. The exception to that general behavior is for the measurements of HernándezÁvila et al. ${ }^{71,95}$ and DeBitetto and Fisher, ${ }^{123}$ which lie closer to our current experimental results, where the differences can increase up to $101 \%$ and $141 \%$, respectively. In our previous investigation using the same set of nitrogen cross sections, we compared the available experimental measurements with our simulated Townsend ionization coefficients calculated using a two-term approximation. Comparing with the two-term simulation results, the overestimation of the experimental measurements of $\alpha_{T} / n_{0}$ reduces to below $28 \%$ over the range of $E / n_{0}$ considered here. For all of the simulated transport coefficients in the present study, a full multiterm treatment is utilized with $l_{\max }$ incremented until convergence is achieved.

We also consider the variation in the calculated transport coefficients to changes in temperatures between $293 \mathrm{~K}$ and $300 \mathrm{~K}$, and the sharing of the postionization energy between the two electrons. In $\mathrm{N}_{2}$, sensitivity to this temperature range is observed to be less than $0.1 \%$ in the drift velocity and mean energy above $1 \mathrm{Td}$, increasing to $3 \%$ at lower fields. The Townsend ionization coefficient is relatively insensitive to this temperature variation above $100 \mathrm{Td}$, with 
differences below $0.01 \%$. That difference increases below this $E / n_{0}$ to be $1.25 \%$ at $60 \mathrm{Td}$. Comparing the $50 \%-50 \%$ energy sharing fraction to both $1 \%-99 \%$ and AFE sharing cases, results in a less than $0.5 \%$ change in the transport coefficients.

\section{REFERENCES}

${ }^{1}$ N. A. Garland, M. J. Brunger, G. Garcia, J. de Urquijo, and R. D. White, Phys. Rev. A 88, 062712 (2013).

${ }^{2}$ M. J. E. Casey, J. de Urquijo, L. N. Serkovic Loli, D. G. Cocks, G. J. Boyle, D. B. Jones, M. J. Brunger, and R. D. White, J. Chem. Phys. 147, 195103 (2017).

${ }^{3}$ M. G. Kong, G. Kroesen, G. Morfill, T. Nosenko, T. Shimizu, J. van Dijk, and J. L. Zimmermann, New J. Phys. 11, 115012 (2009).

${ }^{4}$ M. Laroussi, IEEE Trans. Plasma Sci. 37, 714 (2009).

${ }^{\mathbf{5}}$ T. C. Montie, K. Kelly-Wintenberg, and J. R. Roth, IEEE Trans. Plasma Sci. 28, $41(2000)$.

${ }^{6}$ G. Fridman, M. Peddinghaus, M. Balasubramanian, H. Ayan, A. Fridman, A. Gutsol, and A. Brooks, Plasma Chem. Plasma Process. 26, 425 (2006).

${ }^{7}$ A. V. Nastuta, I. Topala, C. Grigoras, V. Pohoata, and G. Popa, J. Phys. D: Appl. Phys. 44, 105204 (2011)

${ }^{8}$ V. Miller, A. Lin, and A. Fridman, Plasma Chem. Plasma Process. 36, 259 (2016).

${ }^{9}$ B. Boudaïffa, P. Cloutier, D. Hunting, M. A. Huels, and L. Sanche, Science 287, 1658 (2000).

${ }^{10}$ S. Lazović, D. Maletić, A. Leskovac, J. Filipović, N. Puač, G. Malović, G. Joksić, and Z. L. Petrović, Appl. Phys. Lett. 105, 124101 (2014).

${ }^{11}$ M. C. Fuss, L. Ellis-Gibbings, D. B. Jones, M. Brunger, F. Blanco, A. Muñoz, P. Limão-Vieira, and G. Garcia, J. Appl. Phys. 117, 214701 (2015).

${ }^{12}$ J. T. Bushberg, J. A. Siebert, E. M. Leidholdt, Jr., and J. M. Boore, The Essential Physics of Medical Imaging (Lippincott Wiliams \& Wilkins, Philadelphia, 2002).

${ }^{13}$ J. A. LaVerne and S. M. Pimblott, Radiat. Res. 141, 208 (1995).

${ }^{14}$ S. Uehara, H. Nikjoo, and D. T. Goodhead, Radiat. Res. 152, 202 (1999).

${ }^{15}$ S. M. Pimblott and J. A. LaVerne, Radiat. Phys. Chem. 76, 1244 (2007).

${ }^{16}$ L. Sanche, in Radiation Induced Molecular Phenomena in Nucleic Acids, edited by M. K. K. Shukla and J. Leszczynski (Springer, Netherlands, 2008), Vol. 5, p. 531.

${ }^{17}$ V. Cobut, Y. Frongillo, J. P. Patau, T. Goulet, M.-J. Fraser, and J.-P. Jay-Gerin, Radiat. Phys. Chem. 51, 229 (1998).

${ }^{18}$ F. Martin, P. D. Burrow, Z. Cai, P. Cloutier, D. Hunting, and L. Sanche, Phys. Rev. Lett. 93, 068101 (2004).

${ }^{19}$ M. J. Brunger, Int. Rev. Phys. Chem. 36, 333 (2017).

${ }^{20}$ M. U. Bug, W. Yong, H. Rabus, C. Villagrasa, S. Meylan, and A. B. Rosenfeld, Radiat. Phys. Chem. 130, 459 (2017).

${ }^{21}$ E. Alizadeh, T. M. Orlando, and L. Sanche, Annu. Rev. Phys. Chem. 66, 379 (2015).

${ }^{22}$ L. Sanche, Eur. Phys. J. D 35, 367 (2005).

${ }^{23}$ H. Tanaka, M. J. Brunger, L. Campbell, H. Kato, M. Hoshino, and A. R. P. Rau, Rev. Mod. Phys. 88, 025004 (2016).

${ }^{24}$ M. C. Fuss, A. G. Sanz, F. Blanco, P. Limão-Vieira, M. J. Brunger, and G. García, Eur. Phys. J. D 68, 161 (2014).

${ }^{25}$ M. Swadia, Y. Thakar, M. Vinodkumar, and C. Limbachiya, Eur. Phys. J. D 71, 85 (2017).

${ }^{26}$ M. Swadia, R. Bhavsar, Y. Thakar, M. Vinodkumar, and C. Limbachiya, Mol. Phys. 115, 2521 (2017).

${ }^{27}$ R. D. White, M. J. Brunger, N. A. Garland, R. E. Robson, K. F. Ness, G. Garcia, J. de Urquijo, S. Dujko, and Z. L. Petrović, Eur. Phys. J. D 68, 125 (2014).

${ }^{28}$ L. S. Frost and A. V. Phelps, Phys. Rev. 127, 1621 (1962).

${ }^{29}$ A. Engelhardt and A. Phelps, Phys. Rev. 133, A375 (1964)

${ }^{30}$ A. G. Engelhardt, A. V. Phelps, and C. G. Risk, Phys. Rev. 135, A1566 (1964).

${ }^{31}$ L. G. H. Huxley and R. W. Crompton, The Diffusion and Drift of Electrons in Gases (Wiley, New York, 1974).

${ }^{32}$ O. Šašić, J. Jovanović, Z. L. Petrović, J. de Urquijo, J. Castrejón-Pita, J. Hernández-Ávila, and E. Basurto, Phys. Rev. E 71, 046408 (2005).

${ }^{33}$ J. V. Jovanović, E. Basurto, O. Šašić, J. L. Hernández-Ávila, Z. L. Petrović, and J. de Urquijo, J. Phys. D: Appl. Phys. 42, 045202 (2009).
${ }^{34}$ S. Dupljanin, J. de Urquijo, O. Šašić, E. Basurto, A. Juárez, J. Hernández-Ávila, S. Dujko, and Z. L. Petrović, Plasma Sources Sci. Technol. 19, 025005 (2010).

${ }^{35}$ O. Šašić, J. de Urquijo, A. M. Juárez, S. Dupljanin, J. Jovanović, J. L. HernándezÁvila, E. Basurto, and Z. L. Petrović, Plasma Sources Sci. Technol. 19, 034003 (2010).

${ }^{36}$ O. Šašić, S. Dupljanin, J. de Urquijo, and Z. L. Petrović, J. Phys. D: Appl. Phys. 46, 325201 (2013).

${ }^{37}$ G. N. Haddad and T. F. O’Malley, Aust. J. Phys. 35, 35 (1982).

${ }^{38}$ G. N. Haddad, Aust. J. Phys. 36, 297 (1983).

${ }^{39}$ G. N. Haddad, Aust. J. Phys. 37, 487 (1984).

${ }^{40}$ H. B. Milloy, R. W. Crompton, J. A. Rees, and A. G. Robertson, Aust. J. Phys. 30, 61 (1977).

${ }^{41}$ L. G. Christophorou and S. R. Hunter, in Electron Molecule Interactions and Their Applications, edited by L. G. Christophorou (Academic Press, New York, 1984), Vol. 2.

${ }^{42}$ L. G. Christophorou, J. K. Olthoff, and M. V. V. S. Rao, J. Phys. Chem. Ref. Data 25, 1341 (1996).

${ }^{43}$ L. G. Christophorou and J. K. Olthoff, J. Phys. Chem. Ref. Data 28, 967 (1999).

${ }^{44}$ M.-C. Bordage, P. Ségur, L. G. Christophorou, and J. K. Olthoff, J. Appl. Phys. 86, 3558 (1999).

${ }^{45}$ L. G. Christophorou, J. K. Olthoff, and M. Rao, J. Phys. Chem. Ref. Data 26, 1 (1997).

${ }^{46}$ L. G. Christophorou and J. K. Olthoff, J. Phys. Chem. Ref. Data 27, 1 (1998).

${ }^{47}$ L. G. Christophorou and J. K. Olthoff, J. Phys. Chem. Ref. Data 27, 889 (1998).

${ }^{48}$ L. G. Christophorou and J. K. Olthoff, J. Phys. Chem. Ref. Data 29, 267 (2000).

${ }^{49}$ L. G. Christophorou and J. K. Olthoff, J. Phys. Chem. Ref. Data 29, 553 (2000).

${ }^{50}$ R. E. Robson, R. D. White, and K. F. Ness, J. Chem. Phys. 134, 064319 (2011).

${ }^{51}$ K. F. Ness, R. E. Robson, M. J. Brunger, and R. D. White, J. Chem. Phys. 136, 024318 (2012).

${ }^{52}$ J. de Urquijo, E. Basurto, A. M. Juárez, K. F. Ness, R. E. Robson, M. J. Brunger, and R. D. White, J. Chem. Phys. 141, 014308 (2014).

${ }^{53}$ J. L. Hernández-Ávila, E. Basurto, and J. de Urquijo, J. Phys. D: Appl. Phys. 35, 2264 (2002).

${ }^{54}$ E. Basurto, J. L. Hernández-Ávila, A. M. Juárez, and J. de Urquijo, J. Phys. D: Appl. Phys. 46, 355207 (2013).

${ }^{55}$ R. E. Robson, Aust. J. Phys. 44, 685 (1991).

${ }^{56}$ J. Brambring, Z. Phys. 179, 532 (1964).

${ }^{57}$ J. de Urquijo, A. M. Juarez, J. C. Rodríguez-Luna, and J. S. Ramos-Salas, IEEE Trans. Plasma Sci. 35, 1204 (2007).

${ }^{58}$ S. Dujko, R. D. White, and Z. L. Petrović, J. Phys. D: Appl. Phys. 41, 245205 (2008).

${ }^{59}$ R. D. White, R. E. Robson, B. Schmidt, and M. A. Morrison, J. Phys. D: Appl. Phys. 36, 3125 (2003).

${ }^{60}$ B. I. Davydov, Phys. Z. Sowj. Un. 8, 59 (1935).

${ }^{61}$ R. D. White and R. E. Robson, Phys. Rev. E 84, 031125 (2011).

${ }^{62}$ F. B. Pidduck, Q. J. Math. 7, 199 (1936).

${ }^{63}$ I. D. Reid, Aust. J. Phys. 32, 231 (1979).

${ }^{64}$ A. V. Phelps and L. C. Pitchford, Phys. Rev. A 31, 2932 (1985).

${ }^{65}$ T. Makabe and R. White, J. Phys. D: Appl. Phys. 48, 485205 (2015).

${ }^{66}$ K. F. Ness and R. E. Robson, Phys. Rev. A 34, 2185 (1986).

${ }^{67}$ M. J. E. Casey, G. J. Boyle, D. G. Cocks, M. J. Brunger, S. Dujko, J. de Urquijo, and R. D. White, "Thermal induced NDC of electron swarms in $\mathrm{N}_{2}$ and $\mathrm{N}_{2}$-like gases: The role of temperature and collision operator approximations," Plasma Sources Sci. Technol. (to be published); e-print arXiv:1904.06671 [physics.chem-ph].

${ }^{68}$ G. J. Boyle, W. J. Tattersall, D. G. Cocks, S. Dujko, and R. D. White, Phys. Rev. A 91, 052710 (2015).

${ }^{69}$ G. J. Boyle, R. P. McEachran, D. G. Cocks, and R. D. White, J. Chem. Phys. 142, 154507 (2015).

${ }^{70}$ The quasielastic cross section of THF is the combination of the elastic and low-threshold rotational and possibly vibrational processes.

${ }^{71}$ J. L. Hernández-Ávila, E. Basurto, and J. de Urquijo, J. Phys. D: Appl. Phys. 37, 3088 (2004). 
${ }^{72}$ H. V. Duque, T. P. T. Do, M. C. A. Lopes, D. A. Konovalov, R. D. White, M. J. Brunger, and D. B. Jones, J. Chem. Phys. 142, 124307 (2015).

${ }^{73}$ T. P. T. Do, M. Leung, M. Fuss, G. Garcia, F. Blanco, K. Ratnavelu, and M. J. Brunger, J. Chem. Phys. 134, 144302 (2011).

${ }^{74}$ K. Aflatooni, A. M. Scheer, and P. D. Burrow, J. Chem. Phys. 125, 054301 (2006).

${ }^{75}$ R. Janečková, O. May, A. Milosavljević, and J. Fedor, Int. J. Mass Spectrom. 365, 163 (2014).

${ }^{76}$ P. Możejko and L. Sanche, Radiat. Phys. Chem. 73, 77 (2005).

${ }^{77}$ M. Dampc, E. Szymańska, B. Mielewska, and M. Zubek, J. Phys. B: At., Mol. Opt. Phys. 44, 055206 (2011).

${ }^{78}$ M. C. Fuss, R. Colmenares, A. G. Sanz, A. Muñoz, J. C. Oller, F. Blanco, T. P. T. Do, M. J. Brunger, D. Almeida, P. Limão-Vieira, and G. García, J. Phys.: Conf. Ser. 373, 012010 (2012).

${ }^{79}$ M. C. Fuss, A. Muñoz, J. C. Oller, F. Blanco, D. Almeida, P. Limão-Vieira, T. P. D. Do, M. J. Brunger, and G. García, Phys. Rev. A 80, 052709 (2009).

${ }^{80}$ C. J. Colyer, V. Vizcaino, J. P. Sullivan, M. J. Brunger, and S. J. Buckman, New J. Phys. 9, 41 (2007)

${ }^{81}$ A. Gauf, L. Hargreaves, A. Jo, J. Tanner, M. Khakoo, T. Walls, C. Winstead, and V. McKoy, Phys. Rev. A 85, 052717 (2012).

${ }^{82}$ W. Y. Baek, M. Bug, H. Rabus, E. Gargioni, and B. Grosswendt, Phys. Rev. A 86, 032702 (2012)

${ }^{83}$ M. Dampc, A. R. Milosavljević, I. Linert, B. P. Marinković, and M. Zubek, Phys. Rev. A 75, 042710 (2007).

${ }^{84}$ L. Zhang, W. Sun, Y. Zhang, Z. Fan, S. Hu, and Q. Fan, J. Phys. B: At., Mol. Opt. Phys. 50, 085201 (2017).

${ }^{85}$ A. Robertson, Aust. J. Phys. 30, 39 (1977).

${ }^{86}$ J. Pack and A. Phelps, Phys. Rev. 121, 798 (1961).

${ }^{87}$ J. L. Pack and A. V. Phelps, "Dutton database," www.lxcat.net (1961); accessed 03 January 2018.

${ }^{88}$ We note that in measurements of $\alpha_{\text {eff }} / n_{0}$ in mixtures with tetrahydrofurfuryl alcohol (THFA) by the México group, a similar "resonance" feature was present in the very low THFA admixtures in argon, as shown in the work of Foster. In the swarm inversion analysis by our group, we note that the feature necessary in the DEA cross section to reproduce the pure THF $\alpha_{\text {eff }} / n_{0}$ is in very good agreement with the necessary changes to the DEA cross section for THFA to reproduce the THFA-Ar measurements.

${ }^{89}$ S. G. Lias, in NIST Chemistry WebBook: NIST Standard Reference Database Number 69, edited by P. J. Linstrom and W. G. Mallard (National Institute of Standards and Technology, Gaithersburg, MD, 2018); Retrieved April 32019.

${ }^{90}$ A. Goyette, J. de Urquijo, Y. Wang, L. G. Christophorou, and J. K. Olthoff, J. Chem. Phys. 114, 8932 (2001).

${ }^{91}$ A. Bekstein, J. de Urquijo, J. Hernández-Ávila, and E. Basurto, Eur. Phys. J. D 66, 77 (2012).

${ }^{92}$ R. D. White and R. E. Robson, Phys. Rev. Lett. 102, 230602 (2009).

${ }^{93}$ R. D. White, W. J. Tattersall, G. J. Boyle, R. E. Robson, S. Dujko, Z. Lj. Petrović, A. Banković, M. J. Brunger, J. P. Sullivan, S. J. Buckman, and G. García, Appl. Radiat. Isotopes 83, 77 (2014).

${ }^{94}$ Z. L. Petrović, S. Marjanović, S. Dujko, A. Banković, G. Malović, S. Buckman, G. Garcia, R. White, and M. Brunger, Applied Radiation and Isotopes 83, 148 (2014).

${ }^{95}$ J. L. Hernández-Ávila, E. Basurto, and J. de Urquijo, "UNAM database," www.lxcat.net (2004); accessed 03 January 2018.

${ }^{96}$ H. N. Kucukarpaci and J. Lucas, J. Phys. D: Appl. Phys. 14, 2001 (1981).

${ }^{97}$ H. N. Kucukarpaci and J. Lucas, "IST-Lisbon database," www.lxcat.net (1981); accessed 03 January 2018.

${ }^{98}$ Y. Nakamura and M. Kurachi, J. Phys. D: Appl. Phys. 21, 718 (1988).

${ }^{99} \mathrm{D}$. Golden and L. Fisher, Phys. Rev. 123, 1079 (1961).

${ }^{100}$ D. Golden and L. Fisher, "IST-Lisbon database," www.lxcat.net (1961); accessed 03 January 2018.

${ }^{101}$ A. Kruithof, Physica 7, 519 (1940).

${ }^{102}$ A. Kruithof, “IST-Lisbon database," www.lxcat.net (1940); accessed 03 January 2018.
${ }^{103}$ L. Specht, S. Lawton, and T. DeTemple, J. Appl. Phys. 51, 166 (1980).

${ }^{104}$ L. Specht, S. Lawton, and T. DeTemple, "IST-Lisbon database," www.lxcat.net (1980); accessed 03 January 2018.

${ }^{105}$ L. C. Pitchford, L. L. Alves, K. Bartschat, S. F. Biagi, M.-C. Bordage, I. Bray, C. E. Brion, M. J. Brunger, L. Campbell, A. Chachereau et al., Plasma Processes Polym. 14, 1600098 (2017).

${ }^{106}$ J. J. Lowke, Aust. J. Phys. 16, 115 (1963).

${ }^{107}$ Y. Nakamura, J. Phys. D: Appl. Phys. 20, 933 (1987).

${ }^{108}$ W. Fischer-Treuenfeld, Z. Phys. 185, 336 (1965).

${ }^{109}$ W. Fischer-Treuenfeld, “Dutton database," www.lxcat.net (1965); accessed 03 January 2018.

${ }^{110}$ L. Frommhold, Z. Phys. 160, 554 (1960).

${ }^{111}$ L. Frommhold, "Dutton database," www.lxcat.net (1960); accessed 03 January 2018.

${ }^{112}$ A. Prasad and G. Smeaton, Br. J. Appl. Phys. 18, 371 (1967).

${ }^{113}$ A. Prasad and G. Smeaton, "Dutton database," www.lxcat.net (1967); accessed 03 January 2018.

${ }^{114}$ A. B. Wedding, H. A. Blevin, and J. Fletcher, J. Phys. D: Appl. Phys. 18, 2361 (1985).

${ }^{115}$ W. Roznerski, J. Phys. D: Appl. Phys. 29, 614 (1996).

${ }^{116}$ R. A. Snelson and J. Lucas, "Laplace database," www.lxcat.net (1975); accessed 03 January 2018.

${ }^{117}$ L. J. Kelly, Ph.D. thesis, Flinders University of South Australia, 1990.

${ }^{118}$ L. Campbell, M. J. Brunger, A. M. Nolan, L. J. Kelly, A. B. Wedding, J. Harrison, P. J. O. Teubner, D. C. Cartwright, and B. McLaughlin, J. Phys. B: At., Mol. Opt. Phys. 34, 1185 (2001).

${ }^{119}$ F. T. Bagnall and S. C. Haydon, "Dutton database," www.lxcat.net (1965); accessed 03 January 2018.

${ }^{120}$ D. T. A. Blair, "Dutton database," www.lxcat.net (1966); accessed 03 January 2018.

${ }^{121}$ A. H. Cookson, B. W. Ward, and T. J. Lewis, "Dutton database," www.lxcat.net (1966); accessed 03 January 2018.

${ }^{122}$ T. N. Daniel and F. M. Harris, "Dutton database," www.lxcat.net (1970); accessed 03 January 2018.

${ }^{123}$ D. J. DeBitetto and L. H. Fisher, "Dutton database," www.lxcat.net (1956); accessed 03 January 2018.

${ }^{124}$ J. Dutton, S. C. Haydon, and F. Llewellyn-Jones, "Dutton database," www.lxcat.net (1952); accessed 03 January 2018.

${ }^{125}$ M. A. Folkard and S. C. Haydon, "Dutton database," www.lxcat.net (1973); accessed 03 January 2018.

${ }^{126}$ A. E. D. Heylen, “Dutton database," www.lxcat.net (1959); accessed 03 January 2018.

${ }^{127}$ J. Jones, "Dutton database," www.lxcat.net (1968); accessed 03 January 2018.

${ }^{128}$ W. T. McArthur and D. J. Tedford, “Dutton database," www.lxcat.net (1970); accessed 03 January 2018.

${ }^{129}$ B. W. Ward, "Dutton database," www.lxcat.net (1965); accessed 03 January 2018.

${ }^{130}$ S. C. Haydon and O. M. Williams, "LAPLACE database," www.lxcat.net (1976); accessed 03 January 2018.

${ }^{131}$ S. F. Biagi, "Biagi database (Magboltz version 8.97)," www.lxcat.net (2012); accessed 03 January 2018.

${ }^{132}$ S. F. Biagi, "Magboltz version 8.97," http://cern.ch/magboltz, accessed 31 December 2017.

${ }^{133}$ S. Pancheshnyi, S. Biagi, M. Bordage, G. Hagelaar, W. Morgan, A. Phelps, and L. Pitchford, Chem. Phys. 398, 148 (2012).

${ }^{134}$ L. Pitchford, L. Alves, K. Bartschat, S. Biagi, M. Bordage, A. Phelps, C. Ferreira, G. Hagelaar, W. Morgan, S. Pancheshnyi, V. Puech, A. Stauffer, and O. Zatsassrinny, J. Phys. D: Appl. Phys. 46, 334001 (2013).

${ }^{135}$ Y. Itikawa, J. Phys. Chem. Ref. Data 35, 31 (2006).

${ }^{136}$ E. Gerjuoy and S. Stein, Phys. Rev. 97, 1671 (1955).

${ }^{137}$ E. Gerjuoy and S. Stein, Phys. Rev. 98, 1848 (1955). 